\title{
Design, Synthesis, and Antibacterial Evaluation of Novel Ocotillol Derivatives and Their Synergistic Effects with Conventional Antibiotics
}

\author{
Doudou Zhang ${ }^{\dagger}$, Yucheng Cao ${ }^{\dagger}$, Kaiyi Wang ${ }^{\dagger}$, Zhuoyue Shi, Ruodong Wang, Qingguo Meng * and Yi Bi *D \\ School of Pharmacy, Key Laboratory of Molecular Pharmacology and Drug Evaluation, Ministry of Education, \\ Collaborative Innovation Center of Advanced Drug Delivery System and Biotech Drugs in Universities of \\ Shandong, Yantai University, Yantai 264005, China; koikoi1998@yeah.net (D.Z.); cchm1187829@163.com (Y.C.); \\ wangky_1994@163.com (K.W.); shizhuoyue2021@163.com (Z.S.); wangruodong2021@163.com (R.W.) \\ * Correspondence: qinggmeng@163.com (Q.M.); beeyee_413@163.com (Y.B.); \\ Tel.: +86-0535-6706022 (Q.M.); +86-0535-6706285 (Y.B.) \\ + These authors have equally contributed to the work.
}

Citation: Zhang, D.; Cao, Y.; Wang, K.; Shi, Z.; Wang, R.; Meng, Q.; Bi, Y. Design, Synthesis, and Antibacterial Evaluation of Novel Ocotillol Derivatives and Their Synergistic Effects with Conventional Antibiotics. Molecules 2021, 26, 5969. https:// doi.org/10.3390/molecules26195969

Academic Editor: Peter J. Rutledge

Received: 6 September 2021

Accepted: 28 September 2021

Published: 1 October 2021

Publisher's Note: MDPI stays neutral with regard to jurisdictional claims in published maps and institutional affiliations.

Copyright: (c) 2021 by the authors. Licensee MDPI, Basel, Switzerland. This article is an open access article distributed under the terms and conditions of the Creative Commons Attribution (CC BY) license (https:// creativecommons.org/licenses/by/ $4.0 /)$.

\begin{abstract}
The improper use of antibiotics has led to the development of bacterial resistance, resulting in fewer antibiotics for many bacterial infections. Especially, the drug resistance of hospital-acquired methicillin-resistant Staphylococcus aureus (HA-MRSA) is distinctly serious. This research designed and synthesized two series of 3-substituted ocotillol derivatives in order to improve their anti-HAMRSA potency and synergistic antibacterial activity. Among the synthesized compounds, 20-31 showed minimum inhibitory concentration (MIC) values of $1-64 \mu \mathrm{g} / \mathrm{mL}$ in vitro against HA-MRSA 18-19, 18-20, and S. aureus ATCC29213. Compound 21 showed the best antibacterial activity, with an MIC of $1 \mu \mathrm{g} / \mathrm{mL}$ and had synergistic inhibitory effects. The fractional inhibitory concentration index (FICI) value was 0.375 , when combined with chloramphenicol (CHL) or kanamycin (KAN). The structure-activity relationships (SARs) of ocotillol-type derivatives were also summarized. Compound $\mathbf{2 1}$ has the potential to be developed as a novel antibacterial agent or potentiator against HA-MRSA.
\end{abstract}

Keywords: ocotillol-type derivatives; HA-MRSA; antibiotics; structural modification; synergistic effects

\section{Introduction}

Globally, most bacteria (e.g., Mycobacterium tuberculosis, Escherichia coli, and Staphylococcus aureus) can cause severe human diseases (e.g., tuberculosis, sepsis, and skin infections) [1,2]. Because of the improper use and abuse of antibiotics, many bacteria have become resistant or even cross-resistant to many antibacterial drugs on the market, such as methicillin, chloramphenicol (CHL), and kanamycin (KAN) [3,4]. Diseases and deaths caused by drug-resistant bacterial infections have resulted in great losses to human health and property [5]. The World Health Organization prioritized 20 drug-resistant bacteria on the basis of indicators, such as mortality, resistance rates, health care burden, 10-year resistance trends, and transmissibility, which showed that methicillin-resistant Staphylococcus aureus (MRSA) and vancomycin-resistant Enterococcus (VRE) ranked the highest [6].

Natural products are important in the development of new drugs. Over the past four decades, medicinal chemists have become increasingly interested in the development of new drugs derived from natural products $[7,8]$. One of the strategies to treat diseases caused by drug-resistant bacterial infections is to combine existing antibiotics with phytochemicals. Most phytochemicals possess antimicrobial activity and when these plant-derived compounds are used in combination with conventional antibiotics, they can achieve better antibacterial effects and prevent the development of bacterial resistance [9-11]. Betulinic acid isolated from the leaves of Vitex negundo reduces the MIC of methicillin against MRSA 
to $1 / 64 \times$ MIC. When ferulic acid from Phenylpropanoids was combined with amikacin, a synergistic antibacterial effect was noticed against Staphylococcus aureus NCIM 5021 with a fractional inhibitory concentration index (FICI) of 0.16. Epipallocatechin gallate (EGCG), a principal ingredient of tea, drives the anti-MRSA MIC of imipenem from $128 \mu \mathrm{g} / \mathrm{mL}$ to $8 \mu \mathrm{g} / \mathrm{mL}$. Therefore, it is necessary to develop alternative natural or combination drug therapies [12-15] (Figure 1).
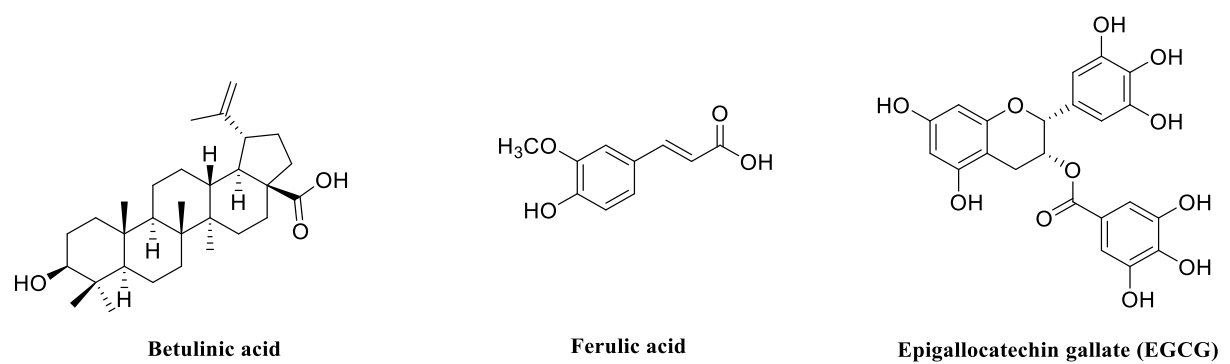

Figure 1. Structures of betulinic acid, ferulic acid, and epipallocatechin gallate (EGCG).

Natural active ingredients derived from terpenoids have good antibacterial activity against MRSA and VRE, such as oleanolic acid (OA) from Salvia officinalis, ursolic acid (UA) from Alstonia scholaris, and 16R-hydroxymollic from Acalypha communis [16,17] (Figure 2). Ginseng is a perennial herb, mainly distributed in China, Russia, and Korea [18]. Ginsenosides and their metabolites (such as G-Rb1, G-Rb2, G-Rd, G-Re, and G-Rg1) have good effects on the human nervous system, cardiovascular system, and endocrine system, among others [18-20]. Ocotillol-type ginsenosides found in Panax quinquefolius L., Vietnamese ginseng, and Panax japonicus have a wide range of excellent pharmacological activities (including anti-ischemic, anti-inflammatory, and antitumor activities) [21]. Our preliminary research found that ocotillol-type ginsenosides have good antibacterial activity, such as (20S, 24S)-ocotillol with an MIC value of $8 \mu \mathrm{g} / \mathrm{mL}$ against MRSA USA300 [22]. After isonipecotic acid modification, compound 1a was synthesized with an MIC value of $8 \mu \mathrm{g} / \mathrm{mL}$ against MRSA USA300. Compound 2a, composed of ocotillol connected with succinic anhydride, had an MIC value of $16 \mu \mathrm{g} / \mathrm{mL}$ against RN4220. Additionally, when compound 1a was combined with KAN, a synergistic antibacterial effect was observed against MRSA USA300 with an FICI of $0.28[23,24]$ (Figure 2).

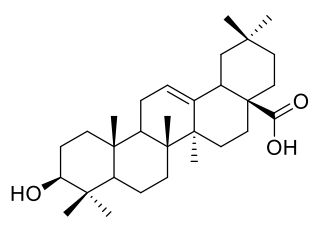

OA

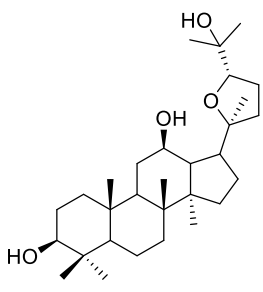

(20S, 24S)-Ocotillol

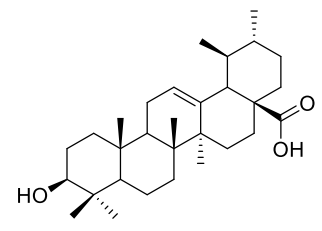

UA

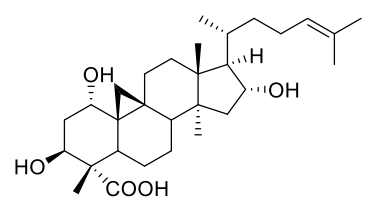

$16 R$-hydroxymollic

Figure 2. Structures of OA, UA, 16R-hydroxymollic, (20S, 24S)-ocotillol, compounds 1a and 2a.

On the basis of the good anti-MRSA potency and synergistic antibacterial activity of the ocotillol-type ginsenosides [25], our group considered $(20 S, 24 S)$-ocotillol and its 
isomer (20S, 24R)-ocotillol as the lead compounds for structural modification. In our previous work, we synthesized different series of ocotillol-type derivatives, from which we identified certain SARs $[22,23,26]$. The antibacterial activity of ocotillol derivatives increases when the pharmacophore contains an amino group or is a hydrogen bond donor [27]. Additionally, studies have shown that nitrogen-containing heterocycles, such as triazoles, morpholines, succinimides, Fmoc-protected amino acids, and dansulfonyl chloride, have extensive and excellent pharmacological activities, especially in terms of their antibacterial properties [28-31]. It is well known that amino acids play important roles in various medical fields, particularly, in the antimicrobial [32]. Researchers have shown that Fmocconjugated amino acids (Fmoc-AA) have a broad range of antimicrobial effects, which linearly correlates with their surfactant property [33]. Therefore, in this research, to obtain compounds with good anti-drug-resistant bacterial potency or synergistic antibacterial activity and further explore the SARs, we synthesized two series of new 3-substituted ocotillol derivatives by introducing nitrogen-containing heterocycles and Fmoc-AA.

Because of the clinical misuse of antibiotics and the higher prevalence of bacteria in hospitals, hospital-acquired methicillin-resistant Staphylococcus aureus (HA-MRSA) spreads faster and is more resistant to marketed antibacterial drugs than common Staphylococcus aureus [34-36]. Therefore, we conducted antibacterial activity assays using S. aureus ATCC 29213, and HA-MRSA strains18-19 and 18-20 on the 18 compounds designed and synthesized in this work so that the experiment was representative of clinical conditions. Furthermore, compounds $\mathbf{2 0}$ and 21, which had good antibacterial activity, were selected for evaluation of their synergistic antibacterial activity with KAN and CHL.

\section{Results and Discussion}

\subsection{Chemistry}

Compounds 4 and 5 were synthesized by a straightforward three-step procedure. The synthesis of ocotillol derivatives $\mathbf{4}$ and $\mathbf{5}$ followed previously reported procedures as shown in Scheme 1.
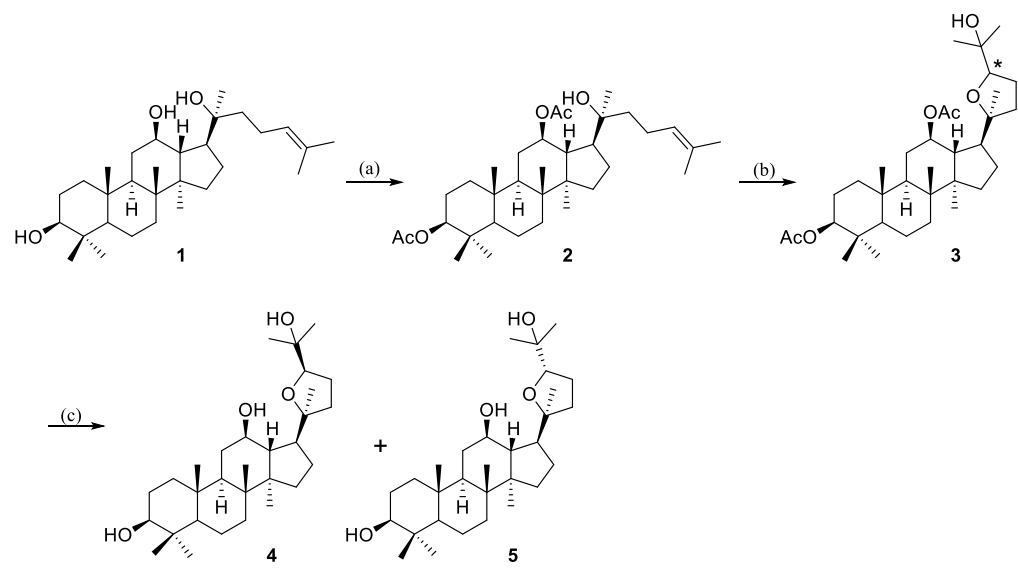

Scheme 1. Synthetic route of ocotillol-type compounds 4 and 5. Reagents and conditions: (a) $\mathrm{Ac}_{2} \mathrm{O}$, DMAP, $\mathrm{CH}_{2} \mathrm{Cl}_{2}$, r.t.; (b) m-CPBA, $\mathrm{CH}_{2} \mathrm{Cl}_{2}$, r.t.; (c) $\mathrm{KOH}, \mathrm{CH}_{3} \mathrm{OH}, 65^{\circ} \mathrm{C}$.

Ocotillol-type derivatives 8-15 were synthesized as shown in Scheme 2. Compound 6 or 7 was obtained by the reaction of compound 4 or $\mathbf{5}$ with succinic anhydride, then compounds 6 and 7 were coupled with different nitrogen-containing heterocycles in dry dichloromethane (DCM) as well as appropriate catalyst to produce compounds 8-15. Ocotillol-type derivatives 20-23, 28-31, 34, and $\mathbf{3 5}$ were synthesized as shown in Scheme 3. Compounds 20-23, 28-31, 34, and 35 were obtained by introducing Fmoc or dansyl chloride through different diamine linkage chains on the basis of compound 4 or 5 . 


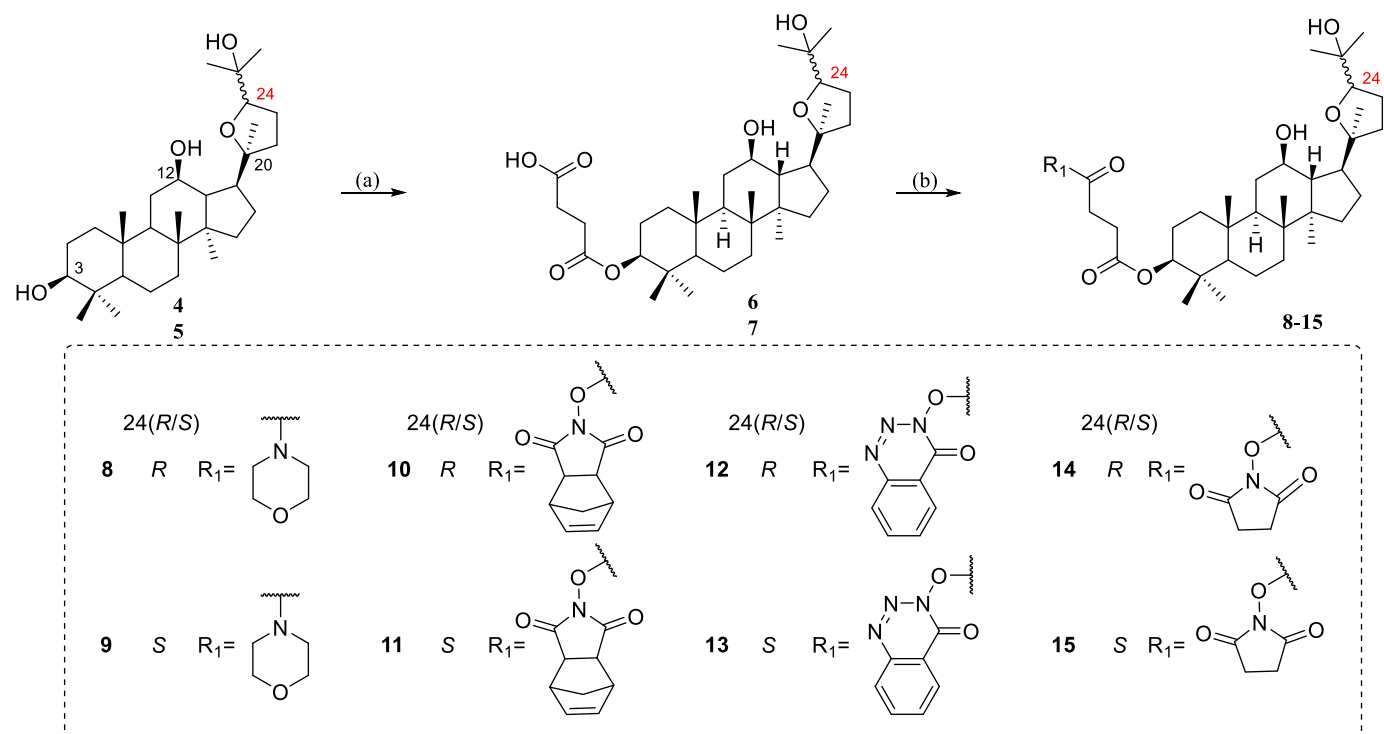

Scheme 2. Synthetic route of ocotillol-type compounds 8-15. Reagents and conditions: (a) Succinic anhydride, DMAP, $\mathrm{CH}_{2} \mathrm{Cl}_{2}$, r.t.; (b) $\mathrm{RH}, \mathrm{EDCI}, \mathrm{CH}_{2} \mathrm{Cl}_{2}$, r.t.
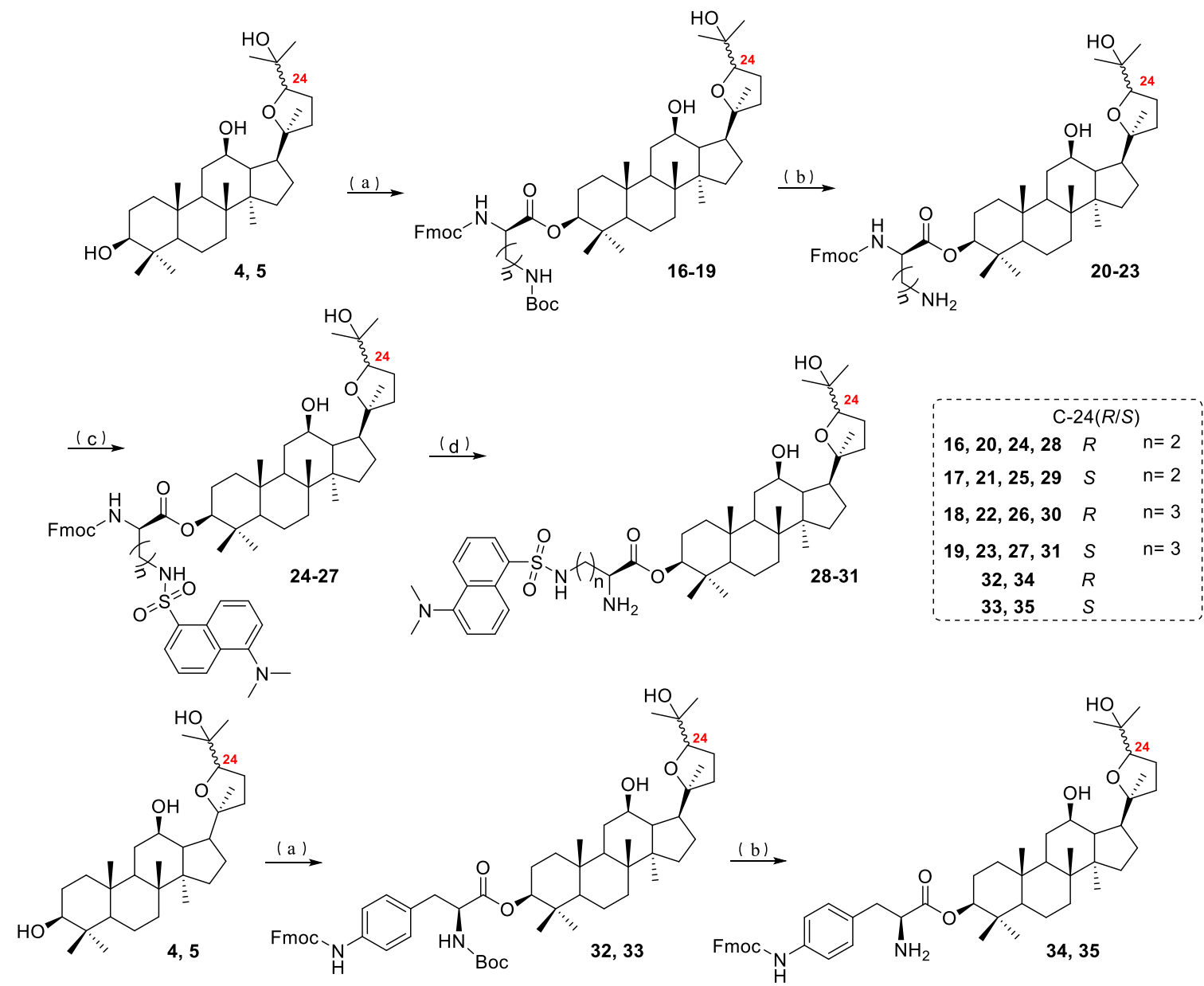

Scheme 3. Synthetic route of ocotillol-type compounds 20-23, 28-31, 34, and 35. Reagents and conditions: (a) DMAP, EDCI, Boc-Fmoc-amino acid, $\mathrm{CH}_{2} \mathrm{Cl}_{2}$, r.t.; (b) TFA, $\mathrm{CH}_{2} \mathrm{Cl}_{2}$, r.t.; (c) DMAP, EDCI, Dansyl chloride, $\mathrm{CH}_{2} \mathrm{Cl}_{2}$, r.t.; (d) piperidine, $\mathrm{CH}_{2} \mathrm{Cl}_{2}$, r.t. 


\subsection{Antibacterial Activity}

The initial antibacterial activity results of the compounds against HA-MRSA 18-19, 18-20, and S. aureus ATCC 29213 are summarized in Table 1. Our previous research showed that ocotillol phthalic anhydride derivatives linked with $N$-containing heterocycles did not demonstrate antibacterial activity. Considering different functional groups and our previous work [24], succinic anhydride was used as a linker. However, the MICs of compounds 8-15 were greater than $128 \mu \mathrm{g} / \mathrm{mL}$, thus the linker had no influence on the antibacterial activity.

Table 1. In vitro antibacterial activity of ocotillol-type derivatives (MIC: $\mu \mathrm{g} / \mathrm{mL}$ ).

\begin{tabular}{cccc}
\hline & \multicolumn{3}{c}{ Antimicrobial Screening MIC $(\mu \mathrm{g} / \mathrm{mL})$} \\
\cline { 2 - 4 } Cpd. & MRSA & MRSA & S. aureus \\
& $\mathbf{1 8 - 1 9}$ & $\mathbf{1 8 - 2 0}$ & ATCC $\mathbf{2 9 2 1 3}$ \\
\hline $\mathbf{8}$ & $>128$ & $>128$ & $>128$ \\
$\mathbf{9}$ & $>128$ & $>128$ & $>128$ \\
$\mathbf{1 0}$ & $>128$ & $>128$ & $>128$ \\
$\mathbf{1 1}$ & $>128$ & $>128$ & $>128$ \\
$\mathbf{1 2}$ & $>128$ & $>128$ & $>128$ \\
$\mathbf{1 3}$ & $>128$ & $>128$ & $>128$ \\
$\mathbf{1 4}$ & $>128$ & $>128$ & $>128$ \\
$\mathbf{1 5}$ & $>128$ & $>128$ & $>128$ \\
$\mathbf{2 0}$ & 4 & 4 & 16 \\
$\mathbf{2 1}$ & 1 & 1 & 1 \\
$\mathbf{2 2}$ & 8 & 2 & 4 \\
$\mathbf{2 3}$ & 4 & 2 & 32 \\
$\mathbf{2 8}$ & 64 & 32 & 32 \\
$\mathbf{2 9}$ & 32 & 64 & 32 \\
$\mathbf{3 0}$ & 4 & 8 & 8 \\
$\mathbf{3 1}$ & 4 & 8 & $>128$ \\
$\mathbf{3 4}$ & $>128$ & $>128$ & $>128$ \\
$\mathbf{3 5}$ & $>128$ & $>128$ & $>128$ \\
$\mathbf{4}$ & $>128$ & $>128$ & 64 \\
$\mathbf{5}$ & 64 & 64 & 0.25 \\
Levofloxacin & 8 & 0.125 & \\
\hline
\end{tabular}

MIC: minimal inhibit concentration.

Among the synthesized compounds, 20-31 inhibited the growth of HA-MRSAs 18-19, 18-20 and S. aureus ATCC 29213 in vitro, with MIC values of 1-64 $\mu \mathrm{g} / \mathrm{mL}$. Compound 21 using $L$-2,4-diaminobutyric acid hydrobromide as the linking chain to the Fmoc group showed better antibacterial activity, with an MIC value of $1 \mu \mathrm{g} / \mathrm{mL}$, while compounds 22 and 23 had good antibacterial activities against HA-MRSA 18-20 with MICs of $2 \mu \mathrm{g} / \mathrm{mL}$. Compared with compounds 20-23, compounds 28-31 showed weaker antibacterial activity. Analysis of the structures of compounds 20-23 and 28-31 indicated that compounds 20-23, which were linked to the Fmoc group, had better antibacterial activity than compounds 28-31, which were linked to the dansyl chloride. The stereochemistry at C-24 dramatically affects the antibacterial activity, with the S-configuration preferred when C-3 hydroxyl is not substituted. However, the dramatic difference in antibacterial activity between the S-configuration and R-configuration caused by the stereochemistry at C-24 seemed to be overpowered by substitutions at C-3 hydroxyl [22,37]. Compounds 20-23 and 28-31, which were substituted with Fmoc or dansyl chloride at the C-3 hydroxyl, showed no significant differences in activity among stereoisomers against the same drug-resistant strain.

Compound 20 and its isomer 21 were chosen for testing against HA-MRSA 18-15, 18-19, 18-20, and S. aureus ATCC 29213 (Figure 3). The result showed that compounds 20 and 21 have good antibacterial activity with an MIC of 4 and $1 \mu \mathrm{g} / \mathrm{mL}$, respectively. It is suggested that the aromatic Fmoc and the length of the primary amine may play vital roles in the binding of the pharmacologically active compounds. On the basis of the results of 
the antibacterial activity and previous research in our laboratory, strain HA-MRSA 18-15 was chosen for assessment of synergistic antibacterial activity.

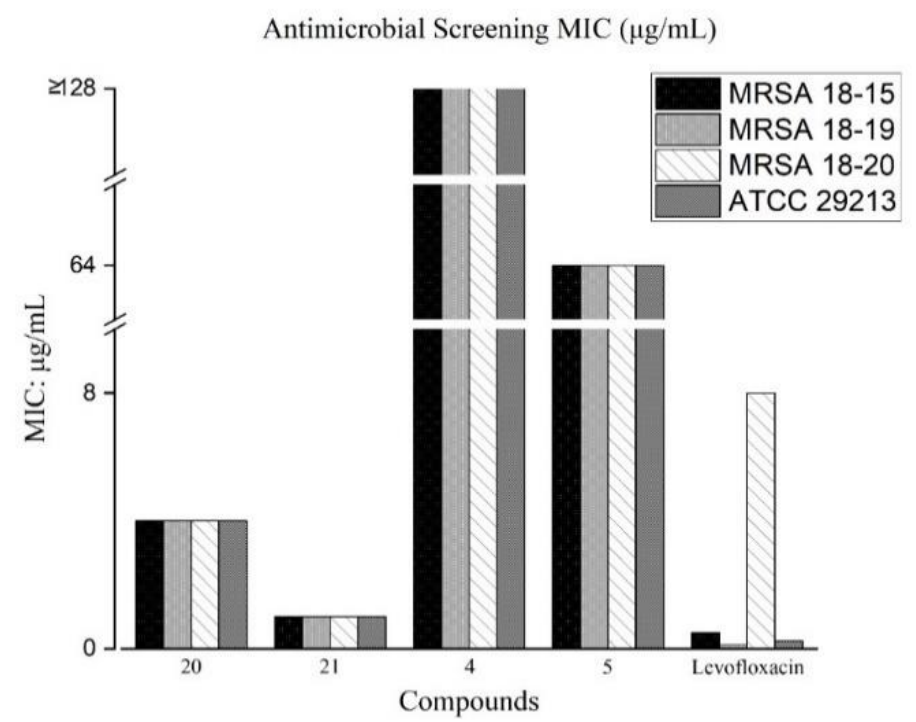

Figure 3. In vitro antibacterial activity of ocotillol-type derivatives (MIC: $\mu \mathrm{g} / \mathrm{mL}$ ).

\subsection{Synergistic Antibacterial Activity}

Antibiotics with different phytochemicals are often used in combination to achieve a synergistic antibacterial effect in the clinical treatment of infections caused by drug-resistant bacteria [38]. According to the results in Tables 2 and 3, compounds 20 and 21 possessed synergistic activity when combined with $\mathrm{KAN}$ or CHL (FICI $<0.5)$. FICI was defined as $\mathrm{FICA}+\mathrm{FICB}=\left(\mathrm{MIC}_{\mathrm{A}+\mathrm{B}} / \mathrm{MIC}_{\mathrm{A}}\right)+\left(\mathrm{MIC}_{\mathrm{A}+\mathrm{B}} / \mathrm{MIC}_{\mathrm{B}}\right)$, and when the value of FICI was less than or equal to 0.5 , compounds $A$ and $B$ were deemed to have a synergistic effect.

Table 2. Synergistic effect of CHL with compounds 20 and 21 against MRSA 18-15.

\begin{tabular}{cccc}
\hline & MIC $(\mu \mathrm{g} / \mathrm{mL})$ & MBC $(\mu \mathrm{g} / \mathrm{mL})$ & FICI \\
\cline { 2 - 4 } & MRSA & MRSA & MRSA \\
& $\mathbf{1 8 - 1 5}$ & $\mathbf{1 8 - 1 5}$ & $\mathbf{1 8 - 1 5}$ \\
\hline CHL & 8 & $>32$ & - \\
$\mathbf{2 0}$ & 4 & $>64$ & - \\
$\mathbf{2 1}$ & 2 & $>128$ & - \\
$\mathbf{2 0}+\mathrm{CHL}$ & 2 & NE & 0.5 \\
$\mathbf{2 1}+\mathrm{CHL}$ & 1 & NE & 0.375 \\
\hline
\end{tabular}

CHL: chloramphenicol; NE: no effect; MIC: minimal inhibit concentration; MBC: minimum bactericidal concentration.; FICI: Fractional inhibitory concentration index.

Table 3. Synergistic effect of KAN with compounds 20 and 21 against MRSA 18-15.

\begin{tabular}{cccc}
\hline & MIC $(\mu \mathrm{g} / \mathrm{mL})$ & MBC $(\mu \mathrm{g} / \mathrm{mL})$ & FICI \\
\cline { 2 - 4 } & MRSA & MRSA & MRSA \\
& $\mathbf{1 8 - 1 5}$ & $\mathbf{1 8 - 1 5}$ & $\mathbf{1 8 - 1 5}$ \\
\hline KAN & 2 & $>8$ & - \\
$\mathbf{2 0}$ & 4 & $>64$ & - \\
$\mathbf{2 1}$ & 1 & $>128$ & - \\
$\mathbf{2 0}+$ KAN & 0.5 & NE & 0.3125 \\
$\mathbf{2 1}+$ KAN & 0.25 & NE & 0.375 \\
\hline
\end{tabular}

CHL: chloramphenicol; NE: no effect; MIC: minimal inhibit concentration; MBC: minimum bactericidal concentration.; FICI: Fractional inhibitory concentration index. 
When compound 21 was used with CHL, the antibacterial activity of CHL against HA-MRSA 18-15 was enhanced from 8 to $1 \mu \mathrm{g} / \mathrm{mL}$, and the FICI value was 0.375 . When compounds 20 and 21 were used with KAN, the antibacterial activity of KAN against HA-MRSA 18-15 was significantly enhanced from 2 to 0.5 and $0.25 \mu \mathrm{g} / \mathrm{mL}$, and the FICI value was 0.3125 and 0.375 , respectively. This result showed that compounds 20 and 21 have good synergistic effects in the presence of KAN or CHL and compound 21 is expected to be developed as a synergistic antibacterial drug in the future, but the drug combination did not produce a bactericidal effect.

\subsection{Structure-Activity Relationships (SARs)}

Based on previous data and our recent work, a more comprehensive structure-activity relationship for ocotillol-type derivatives was obtained as follows (Figure 4). (a) A hydrogen donor at C-3 and C-12 is preferred to maintain the antibacterial activity; (b) decreased antibacterial activity was observed when the functional groups at C-3 and C-12 were ketones; (c) ocotillol-type derivatives coupled with $N$-heterocycles containing a tertiary amine did not improve the antibacterial activity; (d) the stereochemistry at C-24 of ocotillol affected the antibacterial activity slightly when C-3 hydroxyl was substituted with Fmoc or dansyl chloride; (e) the antibacterial activity of ocotillol with a connecting Fmoc was better than those with a connecting dansyl chloride; (f) substitution at the C-3 hydroxyl with a primary amine enhanced the activity against Gram-positive bacteria; and(g) ocotillol-type derivatives with long-chain amino acid substituents at C-3 improved the anti-MRSA ability of KAN and CHL.

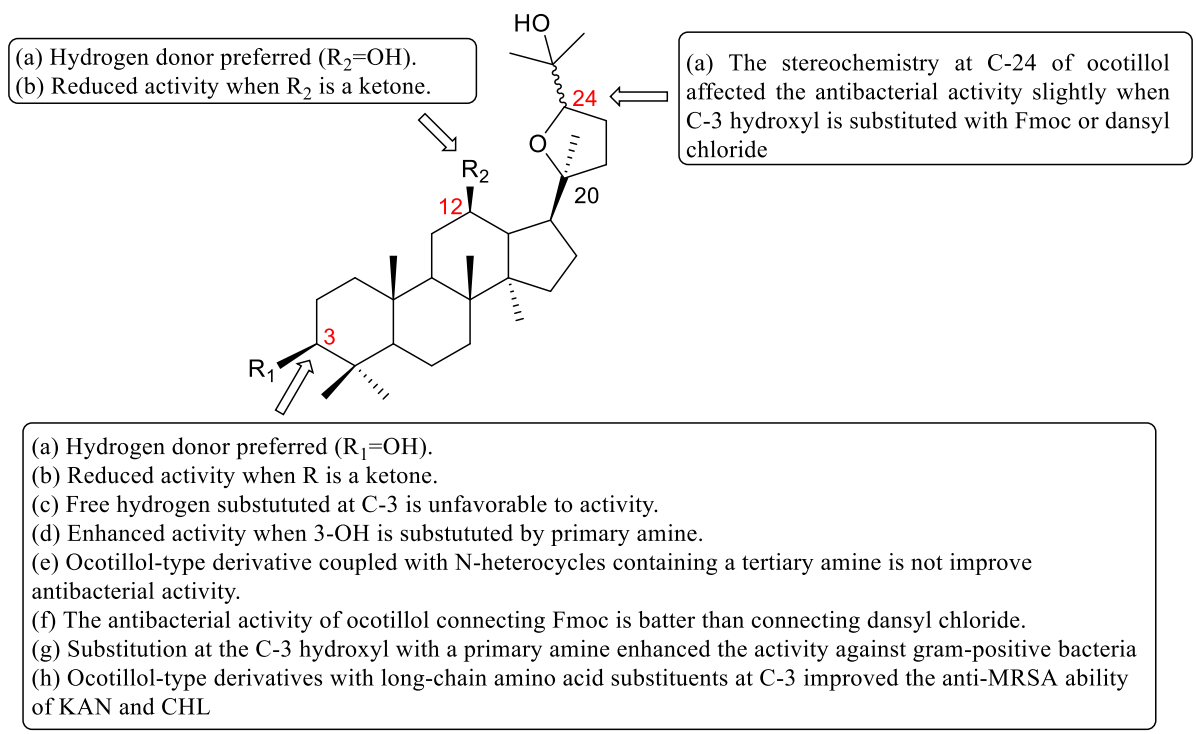

Figure 4. Structure-activity relationships (SARs) of ocotillol-type derivatives.

\section{Materials and Methods}

\subsection{Chemical Reagents and Instruments}

Most of the chemicals and solvents were of analytical grade and the solvents were purified and dried using standard methods. All the structures were verified by nuclear magnetic resonance (NMR), such as ${ }^{1} \mathrm{H}-\mathrm{NMR},{ }^{13} \mathrm{C}-\mathrm{NMR}$, and high-resolution mass spectrometry (HR-MS). Spectra for all the compounds were obtained at $400 \mathrm{MHz}$ for the ${ }^{1} \mathrm{H}$ NMR spectra and at $100 \mathrm{MHz}$ for the ${ }^{13} \mathrm{C}$ NMR using a JNM-ECZ400S (JEOL Ltd., Japan) in $\mathrm{CDCl}_{3}$. HRMS was performed using a Q Exactive TM Orbitrap MS system (Thermo Scientific, USA) in $\mathrm{CH}_{3} \mathrm{OH}$. 


\subsection{The Synthesis of Compounds $\mathbf{8}-\mathbf{1 5}$}

Compound 4 or 5 (500 mg, $1.05 \mathrm{mmol})$, 4-dimethylaminopyridine (383 mg, $3.14 \mathrm{mmol}$ ), and succinic anhydride $(209 \mathrm{mg}, 2.09 \mathrm{mmol})$ were placed in dry dichloromethane $(10 \mathrm{~mL})$, and after stirring at room temperature for $6 \mathrm{~h}$, the resulting mixture was washed with $10 \%$ $\mathrm{HCl}$, water, and brine, dried over anhydrous $\mathrm{Na}_{2} \mathrm{SO}_{4}$, filtered, and evaporated. The white powder intermediate 6 or 7 (542 $\mathrm{mg}, 0.94 \mathrm{mmol})$ was obtained.

A nitrogen-containing heterocycle (morpholine, 3,4-dihydro-3-hydroxy-4-oxo-1,2,3benzotriazine, $\mathrm{N}$-hydroxy-5-norborene-2,3-dicarboximide, or 1-hydroxy-5-pyrrolidinedione) (0.19 mmol), and 1-ethyl-3-(3-dimethylaminopropyl) carbodiimide hydrochloride (EDCI) ( $30 \mathrm{mg}, 0.14 \mathrm{mmol}$ ) was combined with compound 6 (60 mg, $0.10 \mathrm{mmol})$ in anhydrous dichloromethane $(5 \mathrm{~mL})$, and the resulting mixture was stirred for $6 \mathrm{~h}$ at room temperature. The organic solution was washed with water and brine, and dried over anhydrous $\mathrm{Na}_{2} \mathrm{SO}_{4}$. The dichloromethane was evaporated in vacuo and the residue was purified by column chromatography (7:1-3:1 petroleum ether: ethyl acetate) to give the desired products 8-15.

(20S, 24R)-Epoxy-3 $\beta$-O-[2-(1H-morpholin-1-carbonyl) carboxy propionyl]-dammarane$12 \beta, 25$-diol (8)

White solid (yield 73.2\%); m.p. 114.5 115.3 ${ }^{\circ} \mathrm{C}$; $[\alpha] \mathrm{D} 20=41.342\left(\mathrm{c}=0.1, \mathrm{MeOH}: \mathrm{CHCl}_{3}=\right.$ 3:1); ${ }^{1} \mathrm{H}-\mathrm{NMR}\left(\mathrm{CDCl}_{3}, 400 \mathrm{MHz}\right) \delta(\mathrm{ppm}): 4.46(\mathrm{dd}, J=10.7 \mathrm{~Hz}, 5.6 \mathrm{~Hz}, 1 \mathrm{H},-\mathrm{OCH}-), 3.82$ $(\mathrm{dd}, J=8.8 \mathrm{~Hz}, 6.7 \mathrm{~Hz}, 1 \mathrm{H},-\mathrm{OCH}-), 3.71-3.62\left(\mathrm{~m}, 4 \mathrm{H},-\mathrm{CH}_{2}-\times 2\right), 3.59(\mathrm{~d}, J=5.1 \mathrm{~Hz}, 2 \mathrm{H}$, $\left.-\mathrm{CH}_{2}-\right), 3.47\left(\mathrm{q}, J=4.4 \mathrm{~Hz}, 3 \mathrm{H},-\mathrm{CH}_{2}-,-\mathrm{OCH}-\right), 2.64\left(\mathrm{~d}, J=5.2 \mathrm{~Hz}, 2 \mathrm{H},-\mathrm{CH}_{2}-\right), 2.59(\mathrm{~d}$, $\left.J=5.7 \mathrm{~Hz}, 2 \mathrm{H},-\mathrm{CH}_{2}-\right), 1.25\left(\mathrm{~s}, 3 \mathrm{H},-\mathrm{CH}_{3}\right), 1.24\left(\mathrm{~s}, 3 \mathrm{H},-\mathrm{CH}_{3}\right), 1.07\left(\mathrm{~s}, 3 \mathrm{H},-\mathrm{CH}_{3}\right), 0.96(\mathrm{~s}$, $\left.3 \mathrm{H},-\mathrm{CH}_{3}\right), 0.87\left(\mathrm{~s}, 3 \mathrm{H},-\mathrm{CH}_{3}\right), 0.85\left(\mathrm{~s}, 3 \mathrm{H},-\mathrm{CH}_{3}\right), 0.83\left(\mathrm{~s}, 6 \mathrm{H},-\mathrm{CH}_{3} \times 2\right) .{ }^{13} \mathrm{C}-\mathrm{NMR}\left(\mathrm{CDCl}_{3}\right.$, $100 \mathrm{MHz}) \delta$ (ppm): 172.78, 170.05, 86.60, 85.50, 81.09, 71.03, 70.19, 66.95, 66.62, 56.18, 52.10, $50.50,49.47,48.04,45.80,42.15,39.85,38.70,38.01,37.15,34.84,32.69,31.42,31.28,29.66$, 28.67, 28.04, 27.67, 26.21, 25.08, 23.75, 18.24, 16.55, 15.47. HR-MS (ESI) $m / z$ : calcd. for $\mathrm{C}_{38} \mathrm{H}_{63} \mathrm{NO}_{7}[\mathrm{M}+\mathrm{H}]^{+}:$646.46773, found: 646.46735 .

(20S, 24S)-Epoxy-3 $\beta$-O-[2-(1H-morpholin-oxo-carbonyl) carboxy propionyl]-dammarane$12 \beta$, 25-diol (9).

White solid (yield 70.3\%); m.p. 98.5 99.3 $\left.{ }^{\circ} \mathrm{C} ; \alpha\right]_{20}^{D}=39.682\left(\mathrm{c}=0.1, \mathrm{MeOH}: \mathrm{CHCl}_{3}=\right.$ 3:1); ${ }^{1} \mathrm{H}-\mathrm{NMR}\left(\mathrm{CDCl}_{3}, 400 \mathrm{MHz}\right) \delta(\mathrm{ppm}): 4.46(\mathrm{dd}, J=10.6,5.8 \mathrm{~Hz}, 1 \mathrm{H},-\mathrm{OCH}-), 3.84(\mathrm{dd}$, $J=10.8,5.4 \mathrm{~Hz}, 1 \mathrm{H},-\mathrm{OCH}-), 3.64\left(\mathrm{~d}, J=5.1 \mathrm{~Hz}, 4 \mathrm{H},-\mathrm{CH}_{2}-\times 2\right), 3.58(\mathrm{~d}, J=5.1 \mathrm{~Hz}, 2 \mathrm{H}$, $\left.-\mathrm{CH}_{2}-\right), 3.50(\mathrm{dd}, J=10.4,4.8 \mathrm{~Hz}, 1 \mathrm{H},-\mathrm{OCH}-), 3.47\left(\mathrm{~d}, J=5.0 \mathrm{~Hz}, 2 \mathrm{H},-\mathrm{CH}_{2}-\right), 2.64(\mathrm{dd}, J=8.2$, $\left.6.0 \mathrm{~Hz}, 2 \mathrm{H},-\mathrm{CH}_{2}-\right), 2.63-2.55\left(\mathrm{~m}, 2 \mathrm{H},-\mathrm{CH}_{2}-\right), 1.24\left(\mathrm{~s}, 3 \mathrm{H},-\mathrm{CH}_{3}\right), 1.20\left(\mathrm{~s}, 3 \mathrm{H},-\mathrm{CH}_{3}\right), 1.07(\mathrm{~s}$, $\left.3 \mathrm{H},-\mathrm{CH}_{3}\right), 0.98\left(\mathrm{~s}, 3 \mathrm{H},-\mathrm{CH}_{3}\right), 0.88\left(\mathrm{~s}, 6 \mathrm{H},-\mathrm{CH}_{3} \times 2\right), 0.82\left(\mathrm{~s}, 6 \mathrm{H},-\mathrm{CH}_{3} \times 2\right) .{ }^{13} \mathrm{C}-\mathrm{NMR}\left(\mathrm{CDCl}_{3}\right.$, $100 \mathrm{MHz}) \delta$ (ppm): $172.74,170.02,87.45,87.19,81.07,70.55,70.09,66.94,66.60,56.12,52.23$, $50.21,49.00,48.88,45.77,42.14,39.85,38.64,38.02,37.15,34.77,32.28,31.77,29.65,28.95$, $28.61,28.06,27.90,25.13,24.33,23.75,18.25,17.85,16.58,16.40,15.54$. HR-MS (ESI) $m / z$ : calcd. for $\mathrm{C}_{38} \mathrm{H}_{63} \mathrm{NO}_{7}[\mathrm{M}+\mathrm{H}]^{+}$: 646.46773 , found: 646.46692 .

(20S, 24R)-Epoxy-3 $\beta$-O-[2-( $N$-hydroxy-5-norbornene-2,3-dicarboximide-oxo-carbonyl) carboxy propionyl]-dammarane-12 $\beta, 25$-diol (10).

White solid (yield 82.3\%); m.p. 105.9 106.3 ${ }^{\circ} \mathrm{C} ;[\alpha]_{20}^{D}=49.351$ (c $=0.1, \mathrm{MeOH}: \mathrm{CHCl}_{3}=$ 3:1); ${ }^{1} \mathrm{H}-\mathrm{NMR}\left(\mathrm{CDCl}_{3}, 400 \mathrm{MHz}\right) \delta(\mathrm{ppm}): \delta 6.18(\mathrm{~s}, 2 \mathrm{H},-\mathrm{CH}=\mathrm{CH}-), 4.57-4.46(\mathrm{~m}, 1 \mathrm{H}$, -OCH-), $3.84(\mathrm{dd}, J=8.8,6.5 \mathrm{~Hz}, 1 \mathrm{H},-\mathrm{OCH}-), 3.51$ (td, $J=10.5,4.6 \mathrm{~Hz}, 1 \mathrm{H},-\mathrm{OCH}-), 3.44(\mathrm{~s}$, $2 \mathrm{H},-\mathrm{CH}-\times 2), 3.31(\mathrm{~s}, 2 \mathrm{H},-\mathrm{CH}-\times 2), 2.89\left(\mathrm{t}, J=6.1 \mathrm{~Hz}, 2 \mathrm{H},-\mathrm{CH}_{2}-\right), 2.70(\mathrm{t}, J=6.8 \mathrm{~Hz}, 2 \mathrm{H}$, $\left.-\mathrm{CH}_{2}-\right), 1.27\left(\mathrm{~s}, 3 \mathrm{H},-\mathrm{CH}_{3}\right), 1.27\left(\mathrm{~s}, 3 \mathrm{H},-\mathrm{CH}_{3}\right), 1.09\left(\mathrm{~s}, 3 \mathrm{H},-\mathrm{CH}_{3}\right), 0.98\left(\mathrm{~s}, 3 \mathrm{H},-\mathrm{CH}_{3}\right), 0.90$ $\left(\mathrm{s}, 3 \mathrm{H},-\mathrm{CH}_{3}\right), 0.88\left(\mathrm{~s}, 3 \mathrm{H},-\mathrm{CH}_{3}\right), 0.84\left(\mathrm{~s}, 3 \mathrm{H},-\mathrm{CH}_{3}\right), 0.82\left(\mathrm{~s}, 3 \mathrm{H},-\mathrm{CH}_{3}\right) .{ }^{13} \mathrm{C}-\mathrm{NMR}\left(\mathrm{CDCl}_{3}\right.$, $100 \mathrm{MHz}$ ) $\delta$ (ppm): 170.79, 169.90, 134.77, 86.59, 85.47, 81.77, 71.02, 70.21, 56.12, 51.88, 50.47, $49.45,48.03,39.83,38.68,37.97,37.13,34.82,32.68,31.40,31.26,29.17,28.66,28.02,27.65$, 26.20, 25.07, 24.09, 23.63, 21.12, 18.22, 17.44, 16.51, 15.45. HR-MS (ESI) $m / z$ : calcd. for $\mathrm{C}_{43} \mathrm{H}_{63} \mathrm{NO}_{9}[\mathrm{M}+\mathrm{H}]^{+}:$738.45756, found: 738.45691.

(20S, 24S)-Epoxy-3 $\beta$-O-[2-(N-hydroxy-5-norbornene-2,3-dicarboximide-oxo-carbonyl) carboxy propionyl]-dammarane-12 $\beta$, 25-diol (11).

White solid (yield 79.5\%); m.p. 101.8 102.3 ${ }^{\circ} \mathrm{C}$; $[\alpha] \mathrm{D} 20=57.355$ (c = 0.1, $\mathrm{MeOH}: \mathrm{CHCl}_{3}=$ 3:1); ${ }^{1} \mathrm{H}-\mathrm{NMR}\left(\mathrm{CDCl}_{3}, 400 \mathrm{MHz}\right) \delta(\mathrm{ppm}): 6.16(\mathrm{~s}, 2 \mathrm{H},-\mathrm{CH}=\mathrm{CH}-), 4.54-4.46(\mathrm{~m}, 1 \mathrm{H}$, 
-OCH-), 3.86 (dd, J = 10.6, $5.1 \mathrm{~Hz}, 1 \mathrm{H},-\mathrm{OCH}-), 3.56-3.47$ (m, 1H, -OCH-), 3.42 (s, 2H, $-\mathrm{CH}-\times 2), 3.29(\mathrm{~s}, 2 \mathrm{H},-\mathrm{CH}-\times 2), 2.87\left(\mathrm{t}, J=6.6 \mathrm{~Hz}, 2 \mathrm{H},-\mathrm{CH}_{2}-\right), 2.68\left(\mathrm{t}, J=7.3 \mathrm{~Hz}, 2 \mathrm{H},-\mathrm{CH}_{2}^{-}\right)$, $1.25\left(\mathrm{~s}, 3 \mathrm{H},-\mathrm{CH}_{3}\right), 1.21\left(\mathrm{~s}, 3 \mathrm{H},-\mathrm{CH}_{3}\right), 1.08\left(\mathrm{~s}, 3 \mathrm{H},-\mathrm{CH}_{3}\right), 0.99\left(\mathrm{~s}, 3 \mathrm{H},-\mathrm{CH}_{3}\right), 0.89(\mathrm{~s}, 6 \mathrm{H}$, $\left.-\mathrm{CH}_{3} \times 2\right), 0.83\left(\mathrm{~s}, 3 \mathrm{H},-\mathrm{CH}_{3}\right), 0.81\left(\mathrm{~s}, 3 \mathrm{H},-\mathrm{CH}_{3}\right) .{ }^{13} \mathrm{C}-\mathrm{NMR}\left(\mathrm{CDCl}_{3}, 100 \mathrm{MHz}\right) \delta(\mathrm{ppm})$ : $170.73,169.88,134.85,87.48,87.24,81.75,70.59,70.15,56.09,52.24,50.20,48.98,48.86,44.80$, $39.84,37.99,37.15,34.76,32.29,31.72,29.19,28.95,28.62,28.07,28.04,26.41,25.15,24.28$, $21.13,18.25,17.84,16.54,16.39,15.54$. HR-MS (ESI) $m / z$ : calcd. for $\mathrm{C}_{43} \mathrm{H}_{63} \mathrm{NO}_{9}[\mathrm{M}+\mathrm{H}]^{+}$: 738.45756, found: 738.45703 .

(20S, 24R)-Epoxy-3 $\beta$-O-[2-(3-hydroxy-1,2,3-benzotriazin-4(3H)-oxo-carbonyl) carboxy propionyl]-dammarane-12 $\beta, 25-$ diol (12).

Yellow solid (yield 84.1\%); m.p. $154.5 \sim 154.9^{\circ} \mathrm{C}$; $[\alpha]_{20}^{D}=50.020$ (c = 0.1, $\mathrm{MeOH}: \mathrm{CHCl}_{3}$ $=3: 1){ }^{1} \mathrm{H}-\mathrm{NMR}\left(\mathrm{CDCl}_{3}, 400 \mathrm{MHz}\right) \delta(\mathrm{ppm}): 8.39-8.35(\mathrm{~m}, 1 \mathrm{H}, \mathrm{Ar}-\mathrm{H}), 8.22(\mathrm{dt}, J=8.1,0.5 \mathrm{~Hz}$, $1 \mathrm{H}, \mathrm{Ar}-\mathrm{H}), 8.00$ (ddd, $J=8.6,7.3,1.4 \mathrm{~Hz}, 1 \mathrm{H}, \mathrm{Ar}-\mathrm{H}), 7.84$ (ddd, $J=8.4,7.3,0.8 \mathrm{~Hz}, 1 \mathrm{H}, \mathrm{Ar}-\mathrm{H})$, $4.48(\mathrm{dd}, J=10.6,5.6 \mathrm{~Hz}, 1 \mathrm{H},-\mathrm{OCH}-), 3.83$ (dd, $J=8.5,6.7 \mathrm{~Hz}, 1 \mathrm{H},-\mathrm{OCH}-), 3.54-3.49$ (m, $1 \mathrm{H},-\mathrm{OCH}-), 2.62\left(\mathrm{~s}, 4 \mathrm{H},-\mathrm{CH}_{2}-\times 2\right), 1.24\left(\mathrm{~s}, 3 \mathrm{H},-\mathrm{CH}_{3}\right), 1.21\left(\mathrm{~s}, 3 \mathrm{H},-\mathrm{CH}_{3}\right), 1.08\left(\mathrm{~s}, 3 \mathrm{H},-\mathrm{CH}_{3}\right)$, $0.97\left(\mathrm{~s}, 6 \mathrm{H},-\mathrm{CH}_{3} \times 2\right), 0.88\left(\mathrm{~s}, 3 \mathrm{H},-\mathrm{CH}_{3}\right), 0.87\left(\mathrm{~s}, 3 \mathrm{H},-\mathrm{CH}_{3}\right), 0.83\left(\mathrm{~s}, 3 \mathrm{H},-\mathrm{CH}_{3}\right) .{ }^{13} \mathrm{C}-\mathrm{NMR}$ $\left(\mathrm{CDCl}_{3}, 100 \mathrm{MHz}\right) \delta(\mathrm{ppm}): 170.88,168.71,150.27,144.41,135.56,132.87,129.12,125.88$, $122.33,86.61,85.48,81.89,71.04,70.21,60.53,56.11,52.08,51.97,50.45,49.45,48.00,39.80$, $38.65,37.99,37.96,37.12,34.79,32.71,31.29,29.15,28.68,28.02,27.74,26.77,26.22,25.11$, 21.21, 18.28, 16.58, 15.44. HR-MS (ESI) $m / z$ : calcd. for $\mathrm{C}_{41} \mathrm{H}_{59} \mathrm{~N}_{3} \mathrm{O}_{8}[\mathrm{M}+\mathrm{H}]^{+}:$: 22.43749 , found: 722.43677 .

(20S, 24S)-Epoxy-3 $\beta$-O-[2-(3-hydroxy-1,2,3-benzotriazin-4(3H)-oxo-carbonyl) carboxy propionyl]-dammarane-12 $\beta$, 25-diol (13).

Yellow solid (yield 69.3\%); m.p. $149.5 \sim 150.1^{\circ} \mathrm{C} ;[\alpha]_{20}^{D}=31.346$ (c=0.1, $\mathrm{MeOH}: \mathrm{CHCl}_{3}=$ 3:1); ${ }^{1} \mathrm{H}-\mathrm{NMR}\left(\mathrm{CDCl}_{3}, 400 \mathrm{MHz}\right) \delta(\mathrm{ppm}): 8.34(\mathrm{dd}, J=8.0,1.4 \mathrm{~Hz}, 1 \mathrm{H}, \mathrm{Ar}-\mathrm{H}), 8.23-8.18$ (m, 1H, Ar-H), 7.98 (ddd, $J=8.7,7.3,1.4 \mathrm{~Hz}, 1 \mathrm{H}, \mathrm{Ar}-\mathrm{H}), 7.82(\mathrm{ddd}, J=8.4,7.4,1.1 \mathrm{~Hz}, 1 \mathrm{H}$, Ar-H), $4.56-4.50$ (m, 1H, -OCH-), 3.85 (dd, $J=10.8,5.3$ Hz, 1H, -OCH-), 3.51 (td, $J=10.3$, $4.6 \mathrm{~Hz}, 1 \mathrm{H},-\mathrm{OCH}-), 2.86-2.76\left(\mathrm{~m}, 2 \mathrm{H},-\mathrm{CH}_{2}-\right), 2.65-2.55\left(\mathrm{~m}, 2 \mathrm{H},-\mathrm{CH}_{2}-\right), 1.24\left(\mathrm{~s}, 3 \mathrm{H},-\mathrm{CH}_{3}\right)$, $1.20\left(\mathrm{~s}, 3 \mathrm{H},-\mathrm{CH}_{3}\right), 1.07\left(\mathrm{~s}, 3 \mathrm{H},-\mathrm{CH}_{3}\right), 0.98\left(\mathrm{~s}, 3 \mathrm{H},-\mathrm{CH}_{3}\right), 0.88\left(\mathrm{~s}, 6 \mathrm{H},-\mathrm{CH}_{3} \times 2\right), 0.85(\mathrm{~s}, 3 \mathrm{H}$, $\left.-\mathrm{CH}_{3}\right), 0.83\left(\mathrm{~s}, 3 \mathrm{H},-\mathrm{CH}_{3}\right) .{ }^{13} \mathrm{C}-\mathrm{NMR}\left(\mathrm{CDCl}_{3}, 100 \mathrm{MHz}\right) \delta(\mathrm{ppm}): 170.82,168.68,150.25$, $144.40,135.52,132.83,129.10,125.85,122.32,87.47,87.23,81.88,60.49,56.07,52.23,50.18$, $48.93,48.81,39.81,38.61,37.99,37.13,34.73,32.29,31.69,29.16,28.94,28.61,28.07,26.78$, $25.17,24.24,18.24,17.85,16.58,16.40,15.52$. HR-MS (ESI) $m / z$ : calcd. for $\mathrm{C}_{41} \mathrm{H}_{59} \mathrm{~N}_{3} \mathrm{O}_{8}$ $[\mathrm{M}+\mathrm{H}]^{+}:$722.43749, found: 722.43309 .

(20S, 24R)-Epoxy-3 $\beta$-O-[2-(N-hydroxy-succinimide-oxo-carbonyl) carboxy propionyl]damma-rane-12 $\beta$, 25-diol (14).

White solid (yield 82.5\%); m.p. $115.5 \sim 116.3^{\circ} \mathrm{C}$. [ $\left.\alpha\right] \mathrm{D} 20=97.039$ (c=0.1, $\mathrm{MeOH}: \mathrm{CHCl}_{3}=$ 3:1); ${ }^{1} \mathrm{H}-\mathrm{NMR}\left(\mathrm{CDCl}_{3}, 400 \mathrm{MHz}\right) \delta(\mathrm{ppm}): 4.55-4.45$ (m, 1H, -OCH-), 3.83 (dd, J = 8.8, $6.7 \mathrm{~Hz}, 1 \mathrm{H},-\mathrm{OCH}-), 3.49$ (td, $J=10.5,4.5 \mathrm{~Hz}, 1 \mathrm{H},-\mathrm{OCH}-), 2.94(\mathrm{td}, J=7.1,6.7,2.1 \mathrm{~Hz}, 2 \mathrm{H}$, $\left.-\mathrm{CH}_{2}-\right), 2.82\left(\mathrm{~s}, 4 \mathrm{H},-\mathrm{CH}_{2}-\times 2\right), 2.75-2.67\left(\mathrm{~m}, 2 \mathrm{H},-\mathrm{CH}_{2}-\right), 1.26\left(\mathrm{~s}, 3 \mathrm{H},-\mathrm{CH}_{3}\right), 1.25(\mathrm{~s}, 3 \mathrm{H}$, $\left.-\mathrm{CH}_{3}\right), 1.08\left(\mathrm{~s}, 3 \mathrm{H},-\mathrm{CH}_{3}\right), 0.96\left(\mathrm{~s}, 3 \mathrm{H},-\mathrm{CH}_{3}\right), 0.88\left(\mathrm{~s}, 3 \mathrm{H},-\mathrm{CH}_{3}\right), 0.86\left(\mathrm{~s}, 3 \mathrm{H},-\mathrm{CH}_{3}\right), 0.84(\mathrm{~s}$, $\left.3 \mathrm{H},-\mathrm{CH}_{3}\right), 0.82\left(\mathrm{~s}, 3 \mathrm{H},-\mathrm{CH}_{3}\right) .{ }^{13} \mathrm{C}-\mathrm{NMR}\left(\mathrm{CDCl}_{3}, 100 \mathrm{MHz}\right) \delta(\mathrm{ppm}): 170.71,168.92,167.80$, $86.60,85.48,81.85,71.04,70.22,56.14,52.10,50.49,49.46,48.04,39.84,38.69,37.99,37.15$, $34.83,32.69,31.40,31.27,30.65,29.15,28.67,27.98,27.66,26.44,26.21,25.64,25.08,23.66$, 18.24, 16.53, 15.47. HR-MS (ESI) $m / z$ : calcd. for $\mathrm{C}_{38} \mathrm{H}_{59} \mathrm{NO}_{9}[\mathrm{M}+\mathrm{H}]^{+}:$: 74.42626 , found: 674.42621 .

(20S, 24S)-Epoxy-3 $\beta$-O-[2-(N-hydroxy-succinimide-oxo-carbonyl) carboxy propionyl]damma-rane-12 $\beta, 25$-diol (15).

White solid (yield 78.3\%); m.p. $110.2 \sim 110.9^{\circ} \mathrm{C}$. [ $\left.\alpha\right] \mathrm{D} 20=42.692\left(\mathrm{c}=0.1, \mathrm{MeOH}: \mathrm{CHCl}_{3}=\right.$ 3:1); ${ }^{1} \mathrm{H}-\mathrm{NMR}\left(\mathrm{CDCl}_{3}, 400 \mathrm{MHz}\right) \delta(\mathrm{ppm}): 4.54-4.49$ (m, 1H, -OCH-), 3.85 (dd, J = 10.8, $5.4 \mathrm{~Hz}, 1 \mathrm{H},-\mathrm{OCH}-), 3.51$ (td, J = 10.4, $4.9 \mathrm{~Hz}, 1 \mathrm{H},-\mathrm{OCH}-), 2.94\left(\mathrm{t}, \mathrm{J}=7.4 \mathrm{~Hz}, 2 \mathrm{H},-\mathrm{CH}_{2}-\right), 2.81$ $\left(\mathrm{s}, 4 \mathrm{H},-\mathrm{CH}_{2}-\times 2\right), 2.72\left(\mathrm{t}, J=6.8 \mathrm{~Hz}, 2 \mathrm{H},-\mathrm{CH}_{2}-\right), 1.25\left(\mathrm{~s}, 3 \mathrm{H},-\mathrm{CH}_{3}\right), 1.21\left(\mathrm{~s}, 3 \mathrm{H},-\mathrm{CH}_{3}\right), 1.08$ $\left(\mathrm{s}, 3 \mathrm{H},-\mathrm{CH}_{3}\right), 0.99\left(\mathrm{~s}, 3 \mathrm{H},-\mathrm{CH}_{3}\right), 0.89\left(\mathrm{~s}, 6 \mathrm{H},-\mathrm{CH}_{3} \times 2\right), 0.84\left(\mathrm{~s}, 3 \mathrm{H},-\mathrm{CH}_{3}\right), 0.82\left(\mathrm{~s}, 3 \mathrm{H},-\mathrm{CH}_{3}\right)$. ${ }^{13} \mathrm{C}-\mathrm{NMR}\left(\mathrm{CDCl}_{3}, 100 \mathrm{MHz}\right) \delta$ (ppm): 170.69, 168.94, 167.81, 87.46, 87.22, 81.84, 70.55, 70.13, 
$60.47,56.09,52.23,50.20,48.89,39.84,38.63,37.99,37.15,34.76,32.28,31.70,29.14,28.96$, $28.62,28.06,26.44,25.64,25.14,24.31,23.67,18.25,17.84,16.55,16.39,15.54$. HR-MS (ESI) $\mathrm{m} / \mathrm{z}$ : calcd. for $\mathrm{C}_{38} \mathrm{H}_{59} \mathrm{NO}_{9}[\mathrm{M}+\mathrm{H}]^{+}$: 674.42626 , found: 674.42548 .

\subsection{The Synthesis of Compounds 20-23, 28-31, 34, and $\mathbf{3 5}$}

Compound 4 or 5 ( $80 \mathrm{mg}, 0.17 \mathrm{mmol})$ was added to anhydrous dichloromethane $(4 \mathrm{~mL})$, then a Boc-Fmoc-protected amino acid $(0.25 \mathrm{mmol})$, DMAP (56 mg, $0.50 \mathrm{mmol})$, and EDCI (96 mg, $0.50 \mathrm{mmol}$ ) were added slowly. After stirring at room temperature for $3 \mathrm{~h}$, the resulting mixture was washed with $10 \% \mathrm{HCl}$, water, and brine, and dried over anhydrous $\mathrm{Na}_{2} \mathrm{SO}_{4}$. The dichloromethane was evaporated in vacuo to obtain the intermediates 16-19, 32, and 33. Trifluoroacetic acid ( $1 \mathrm{~mL}, 13.43 \mathrm{mmol})$ was added slowly to a solution of each intermediate 16-19, 32, and $33(0.17 \mathrm{mmol})$ in anhydrous dichloromethane $(4 \mathrm{~mL})$. After stirring at room temperature for $2 \mathrm{~h}$, the resulting mixture was filtered and evaporated to obtain the target compounds 20-23, 34, and 35. For compounds 20-23, each compound $(0.17 \mathrm{mmol})$, dansyl chloride $(55 \mathrm{mg}, 0.20 \mathrm{mmol})$, and $N, N$-diisopropylethylamine $(106 \mu \mathrm{L}, 0.65 \mathrm{mmol})$ were combined in anhydrous dichloromethane $(4 \mathrm{~mL})$. After stirring at room temperature for $2 \mathrm{~h}$, the resulting mixture was washed with $5 \% \mathrm{HCl}$, water, and brine, dried over in anhydrous $\mathrm{Na}_{2} \mathrm{SO}_{4}$, filtered, and evaporated to provide intermediates 24-27 (Scheme 3). Compounds 28-31 were synthesized by adding intermediates 24-27 $(0.17 \mathrm{mmol})$ and piperidine $(1 \mathrm{~mL}, 10.88 \mathrm{mmol})$ to anhydrous dichloromethane $(4 \mathrm{~mL})$. After stirring at room temperature for $4 \mathrm{~h}$, the resulting mixture was washed with water and brine, dried over anhydrous $\mathrm{Na}_{2} \mathrm{SO}_{4}$, filtered, and evaporated. The residue was purified over silica gel with chloroform: methanol (100:1-50:1) to provide compounds 28-31.

3.3.1. (20S, 24R)-Epoxy-3ß-O-[2-( $N^{\prime}$-Fmoc)-5-amino butyryl]-dammarane-12 $\beta$, 25-diol (20)

White solid (yield 77.4\%); m.p. $174.2 \sim 174.7^{\circ} \mathrm{C} ;[\alpha]_{20}^{D}=108.370\left(\mathrm{c}=0.1, \mathrm{MeOH}: \mathrm{CHCl}^{3}=3: 1\right)$; ${ }^{1} \mathrm{H}-\mathrm{NMR}\left(\mathrm{CDCl}_{3}, 400 \mathrm{MHz}\right) \delta(\mathrm{ppm}): 8.27\left(\mathrm{~s}, 2 \mathrm{H},-\mathrm{NH}_{2}\right), 7.70(\mathrm{~d}, J=7.6 \mathrm{~Hz}, 2 \mathrm{H}, \mathrm{Ar}-\mathrm{H} \times 2)$, $7.55(\mathrm{~d}, J=6.6 \mathrm{~Hz}, 2 \mathrm{H}, \mathrm{Ar}-\mathrm{H} \times 2), 7.34(\mathrm{t}, J=7.4 \mathrm{~Hz}, 2 \mathrm{H}, \mathrm{Ar}-\mathrm{H} \times 2), 7.26(\mathrm{t}, J=7.5 \mathrm{~Hz}, 2 \mathrm{H}$, $\mathrm{Ar}-\mathrm{H} \times 2), 6.20$ (d, J = 7.4 Hz, 1H, -NH-), 4.47 (d, J = 7.3 Hz, 1H, -OCH-), 4.32 (d, J = 6.5 Hz, $\left.3 \mathrm{H},-\mathrm{OCH}_{2^{-}},-\mathrm{NCH}-\right), 4.14(\mathrm{t}, J=6.8 \mathrm{~Hz}, 1 \mathrm{H},-\mathrm{CH}-), 3.81(\mathrm{t}, J=7.6 \mathrm{~Hz}, 1 \mathrm{H},-\mathrm{OCH}-), 3.60-$ 3.47 (m, 1H, -OCH-), 3.04 (d, J = 43.0 Hz, 2H, $\left.-\mathrm{NCH}_{2}-\right), 2.23$ (d, J = 55.9 Hz, 2H, - $\left.\mathrm{CH}_{2}-\right), 1.26$ $\left(\mathrm{s}, 3 \mathrm{H},-\mathrm{CH}_{3}\right), 1.24\left(\mathrm{~s}, 3 \mathrm{H},-\mathrm{CH}_{3}\right), 1.22\left(\mathrm{~s}, 3 \mathrm{H},-\mathrm{CH}_{3}\right), 1.09\left(\mathrm{~s}, 3 \mathrm{H},-\mathrm{CH}_{3}\right), 0.94\left(\mathrm{~s}, 3 \mathrm{H},-\mathrm{CH}_{3}\right)$, $0.86\left(\mathrm{~s}, 3 \mathrm{H},-\mathrm{CH}_{3}\right), 0.82\left(\mathrm{~s}, 3 \mathrm{H},-\mathrm{CH}_{3}\right), 0.79\left(\mathrm{~s}, 3 \mathrm{H},-\mathrm{CH}_{3}\right) .{ }^{13} \mathrm{C}-\mathrm{NMR}\left(\mathrm{CDCl}_{3}, 100 \mathrm{MHz}\right) \delta$ (ppm): 171.18, 157.09, 143.75, 141.32, 132.50, 131.01, 128.89, 127.82, 127.21, 125.20, 120.04, $86.52,85.24,83.25,71.13,70.42,68.24,67.47,56.08,52.10,51.59,50.35,49.28,48.07,47.08$, $39.78,38.79,38.48,37.95,37.06,34.75,32.52,32.03,31.36,31.11,29.80,29.01,28.01,27.48$, 26.10, 25.09, 23.81, 23.35, 23.09, 22.80, 18.17, 16.25, 15.47. HR-MS (ESI) $\mathrm{m} / \mathrm{z}$ : calcd. for $\mathrm{C}_{49} \mathrm{H}_{70} \mathrm{~N}_{2} \mathrm{O}_{7}[\mathrm{M}+\mathrm{H}]^{+}:$799.52558, found: 799.52283 .

3.3.2. (20S, 24S)-Epoxy-3 $\beta$-O-[2-(N'-Fmoc)-5-amino butyryl]-dammarane-12 $\beta$, 25-diol (21)

White solid (yield 70.1\%); m.p. $191.1 \sim 191.5^{\circ} \mathrm{C}$; $[\alpha]_{20}^{D}=48.686$ (c=0.1, $\mathrm{MeOH}: \mathrm{CHCl}_{3}=$ 3:1); ${ }^{1} \mathrm{H}-\mathrm{NMR}\left(\mathrm{CDCl}_{3}, 400 \mathrm{MHz}\right) \delta(\mathrm{ppm}): 8.24\left(\mathrm{~s}, 2 \mathrm{H},-\mathrm{NH}_{2}\right), 7.69$ (d, J = $7.5 \mathrm{~Hz}, 2 \mathrm{H}, \mathrm{Ar}-$ $\mathrm{H} \times 2), 7.54(\mathrm{~d}, J=5.6 \mathrm{~Hz}, 2 \mathrm{H}, \mathrm{Ar}-\mathrm{H} \times 2), 7.34(\mathrm{t}, J=7.4 \mathrm{~Hz}, 2 \mathrm{H}, \mathrm{Ar}-\mathrm{H} \times 2), 7.25(\mathrm{t}, J=7.4 \mathrm{~Hz}$, $3 \mathrm{H}, \mathrm{Ar}-\mathrm{H} \times 2), 6.13$ (d, J = 7.5 Hz, 1H, -NH-), $4.55-4.43$ (m, 1H, -OCH-), $4.40-4.26$ (m, $\left.3 \mathrm{H},-\mathrm{OCH}_{2-},-\mathrm{NCH}-\right), 4.13(\mathrm{t}, J=6.9 \mathrm{~Hz}, 1 \mathrm{H},-\mathrm{CH}-), 3.92-3.79(\mathrm{~m}, 1 \mathrm{H},-\mathrm{OCH}-), 3.50(\mathrm{~s}, 1 \mathrm{H}$, $-\mathrm{OCH}-), 3.02\left(\mathrm{~d}, \mathrm{~J}=55.7 \mathrm{~Hz}, 2 \mathrm{H},-\mathrm{NCH}_{2}-\right), 2.26\left(\mathrm{~d}, \mathrm{~J}=41.9 \mathrm{~Hz}, 2 \mathrm{H},-\mathrm{CH}_{2}-\right), 1.24\left(\mathrm{~s}, 3 \mathrm{H},-\mathrm{CH}_{3}\right)$, $1.23\left(\mathrm{~s}, 3 \mathrm{H},-\mathrm{CH}_{3}\right), 1.18\left(\mathrm{~s}, 3 \mathrm{H},-\mathrm{CH}_{3}\right), 1.10\left(\mathrm{~s}, 3 \mathrm{H},-\mathrm{CH}_{3}\right), 0.96\left(\mathrm{~s}, 3 \mathrm{H},-\mathrm{CH}_{3}\right), 0.85(\mathrm{~s}, 3 \mathrm{H}$, -CH3), $0.84(\mathrm{~s}, 3 \mathrm{H},-\mathrm{CH} 3), 0.79\left(\mathrm{~s}, 3 \mathrm{H},-\mathrm{CH}_{3}\right)$. 13C-NMR (CDCl3, $\left.100 \mathrm{MHz}\right) \delta(\mathrm{ppm}): 171.13$, 157.02, 143.70, 141.32, 127.81, 127.22, 125.29, 125.27, 125.17, 120.03, 87.66, 87.19, 83.26, 70.66, $67.45,64.51,61.00,55.99,52.19,51.54,50.15,48.87,48.71,47.07,46.29,39.76,39.18,38.47$, $37.92,37.04,36.61,34.67,32.36,32.03,31.69,30.22,29.80,29.47,28.91,28.55,27.99,25.53$, $23.75,23.46,22.80,18.19,16.28,15.47$. HR-MS (ESI) $m / z$ : calcd. for $\mathrm{C}_{49} \mathrm{H}_{70} \mathrm{~N}_{2} \mathrm{O}_{7}[\mathrm{M}+\mathrm{H}]^{+}$: 799.52558, found: 799.52307 . 
3.3.3. (20S, 24R)-Epoxy-3 $\beta$-O-[2-( $N^{\prime}$-Fmoc)-6-amino ornithyl]-dammarane-12 $\beta$, 25-diol (22)

White solid (yield 69.9\%); m.p. 183.5 184.1 ${ }^{\circ} \mathrm{C} ;[\alpha]_{20}^{D}=34.345$ (c $=0.1, \mathrm{MeOH}: \mathrm{CHCl}_{3}=$ 3:1); ${ }^{1} \mathrm{H}-\mathrm{NMR}\left(\mathrm{CDCl}_{3}, 400 \mathrm{MHz}\right) \delta(\mathrm{ppm}): 8.01\left(\mathrm{~s}, 2 \mathrm{H},-\mathrm{NH}_{2}\right), 7.69(\mathrm{~d}, J=7.5 \mathrm{~Hz}, 2 \mathrm{H}, \mathrm{Ar}-$ $\mathrm{H} \times 2), 7.54(\mathrm{t}, J=7.0 \mathrm{~Hz}, 2 \mathrm{H}, \mathrm{Ar}-\mathrm{H} \times 2), 7.32(\mathrm{t}, J=7.5 \mathrm{~Hz}, 2 \mathrm{H}, \mathrm{Ar}-\mathrm{H} \times 2), 7.24(\mathrm{~d}, J=5.7 \mathrm{~Hz}$, $2 \mathrm{H}, \mathrm{Ar}-\mathrm{H} \times 2), 5.84(\mathrm{~d}, J=8.0 \mathrm{~Hz}, 1 \mathrm{H},-\mathrm{NH}-), 4.50-4.38(\mathrm{~m}, 1 \mathrm{H},-\mathrm{OCH}-), 4.27(\mathrm{~d}, J=6.8 \mathrm{~Hz}$, $\left.3 \mathrm{H},-\mathrm{OCH}_{2-},-\mathrm{NCH}-\right), 4.12(\mathrm{t}, J=7.0 \mathrm{~Hz}, 1 \mathrm{H},-\mathrm{CH}-), 3.86-3.77(\mathrm{~m}, 1 \mathrm{H},-\mathrm{OCH}-), 3.48(\mathrm{dd}$, $\mathrm{J}=10.2,4.1 \mathrm{~Hz}, 1 \mathrm{H},-\mathrm{OCH}-), 2.95\left(\mathrm{~s}, 2 \mathrm{H},-\mathrm{NCH}_{2}-\right), 1.25\left(\mathrm{~s}, 3 \mathrm{H},-\mathrm{CH}_{3}\right), 1.24(\mathrm{~s}, 6 \mathrm{H},-\mathrm{CH} 3 \times 2)$, $1.08\left(\mathrm{~s}, 3 \mathrm{H},-\mathrm{CH}_{3}\right), 0.93\left(\mathrm{~s}, 3 \mathrm{H},-\mathrm{CH}_{3}\right), 0.83\left(\mathrm{~s}, 3 \mathrm{H},-\mathrm{CH}_{3}\right), 0.79\left(\mathrm{~s}, 3 \mathrm{H},-\mathrm{CH}_{3}\right), 0.75(\mathrm{~s}, 3 \mathrm{H}$, - $\left.\mathrm{CH}_{3}\right) .{ }^{13} \mathrm{C}-\mathrm{NMR}\left(\mathrm{CDCl}_{3}, 100 \mathrm{MHz}\right) \delta(\mathrm{ppm}): 171.81,156.55,143.95,143.72,141.28,127.78$, $127.18,125.31,125.15,120.01,86.58,85.40,82.84,71.00,70.29,55.98,52.05,50.39,49.38,48.02$, $47.05,39.79,39.36,37.05,34.75,32.65,32.01,31.40,31.23,29.78,29.44,28.66,27.98,27.59$, $26.15,23.74,23.62,22.77,18.18,16.49,16.36,15.43$. HR-MS (ESI) $m / z$ : calcd. for $\mathrm{C}_{50} \mathrm{H}_{72} \mathrm{~N}_{2} \mathrm{O}_{7}$ $[\mathrm{M}+\mathrm{H}]^{+}:$813.54123, found: 813.54254 .

3.3.4. (20S, 24S)-Epoxy-3 $\beta$-O-[2-( $N^{\prime}$-Fmoc)-6-amino ornithyl]-dammarane-12 $\beta$, 25-diol (23)

White solid (yield 67.9\%); m.p. 177.2 177.9 ${ }^{\circ} \mathrm{C} ;[\alpha]_{20}^{D}=96.034$ (c = 0.1, $\mathrm{MeOH}: \mathrm{CHCl}_{3}=$ 3:1); ${ }^{1} \mathrm{H}-\mathrm{NMR}(\mathrm{CDCl} 3,400 \mathrm{MHz}) \delta(\mathrm{ppm}): 8.07\left(\mathrm{~s}, 2 \mathrm{H},-\mathrm{NH}_{2}\right), 7.69(\mathrm{~d}, J=7.5 \mathrm{~Hz}, 2 \mathrm{H}$, $\mathrm{Ar}-\mathrm{H} \times 2), 7.55(\mathrm{t}, J=6.3 \mathrm{~Hz}, 2 \mathrm{H}, \mathrm{Ar}-\mathrm{H} \times 2), 7.33(\mathrm{t}, J=7.5 \mathrm{~Hz}, 2 \mathrm{H}, \mathrm{Ar}-\mathrm{H} \times 2), 7.24(\mathrm{t}, J=7.4 \mathrm{~Hz}$, $2 \mathrm{H}, \mathrm{Ar}-\mathrm{H} \times 2), 5.83(\mathrm{~d}, J=8.0 \mathrm{~Hz}, 1 \mathrm{H},-\mathrm{NH}-), 4.47(\mathrm{t}, J=8.7 \mathrm{~Hz}, 1 \mathrm{H},-\mathrm{OCH}-), 4.28(\mathrm{~d}, J=6.6 \mathrm{~Hz}$, $\left.3 \mathrm{H},-\mathrm{OCH}_{2-},-\mathrm{NCH}-\right), 4.13(\mathrm{t}, J=7.1 \mathrm{~Hz}, 1 \mathrm{H},-\mathrm{CH}-), 3.90-3.81(\mathrm{~m}, 1 \mathrm{H},-\mathrm{OCH}-), 3.54-3.44$ $(\mathrm{m}, 1 \mathrm{H},-\mathrm{OCH}-), 2.97\left(\mathrm{~s}, 2 \mathrm{H},-\mathrm{NCH}_{2}-\right), 1.24\left(\mathrm{~s}, 3 \mathrm{H},-\mathrm{CH}_{3}\right), 1.19\left(\mathrm{~s}, 3 \mathrm{H},-\mathrm{CH}_{3}\right), 1.09(\mathrm{~s}, 3 \mathrm{H}$, $\left.-\mathrm{CH}_{3}\right), 0.96\left(\mathrm{~s}, 3 \mathrm{H},-\mathrm{CH}_{3}\right), 0.85\left(\mathrm{~s}, 3 \mathrm{H},-\mathrm{CH}_{3}\right), 0.84\left(\mathrm{~s}, 3 \mathrm{H},-\mathrm{CH}_{3}\right), 0.77\left(\mathrm{~s}, 3 \mathrm{H},-\mathrm{CH}_{3}\right), 0.76(\mathrm{~s}$, $3 \mathrm{H},-\mathrm{CH} 3) .{ }^{13} \mathrm{C}-\mathrm{NMR}\left(\mathrm{CDCl}_{3}, 100 \mathrm{MHz}\right) \delta$ (ppm): 171.76, 156.54, 143.95, 143.74, 141.29 , $127.78,127.18,125.32,125.18,120.01,87.60,87.22,82.83,70.66,70.43,67.41,58.42,55.94$, $53.85,52.21,50.17,48.93,48.75,47.06,39.77,39.38,38.52,37.96,37.07,34.69,32.32,31.76$, 29.78, 28.90, 28.56, 28.01, 27.84, 25.41, 24.01, 18.17, 16.53, 16.31, 15.49. HR-MS (ESI) $m / z$ : calcd. for $\mathrm{C}_{50} \mathrm{H}_{72} \mathrm{~N}_{2} \mathrm{O}_{7}[\mathrm{M}+\mathrm{H}]^{+}$: 813.54123, found: 813.54169.

3.3.5. (20S, 24R)-Epoxy-3 $\beta-O-[2$-amino-(1-dansylamino)-5-dimethyl amino butyr-yl]-dammarane-12 $\beta$, 25-diol (28)

Yellow solid (yield 72.2\%); m.p. 202.0 202.7 ${ }^{\circ} \mathrm{C} ;[\alpha]_{20}^{D}=51.689$ (c = 0.1, $\mathrm{MeOH}: \mathrm{CHCl}_{3}=$ 3:1); ${ }^{1} \mathrm{H}-\mathrm{NMR}(\mathrm{CDCl} 3,400 \mathrm{MHz}) \delta(\mathrm{ppm}): 8.50(\mathrm{dt}, J=8.5,1.0 \mathrm{~Hz}, 1 \mathrm{H}, \mathrm{Ar}-\mathrm{H}), 8.27(\mathrm{~d}$, $J=8.7 \mathrm{~Hz}, 1 \mathrm{H}, \mathrm{Ar}-\mathrm{H}), 8.22(\mathrm{dd}, J=7.3,1.3 \mathrm{~Hz}, 1 \mathrm{H}, \mathrm{Ar}-\mathrm{H}), 7.51(\mathrm{ddd}, J=14.7,8.6,7.4 \mathrm{~Hz}, 2 \mathrm{H}$, Ar-H×2), $7.16(\mathrm{dd}, J=7.6,0.7 \mathrm{~Hz}, 1 \mathrm{H}, \mathrm{Ar}-\mathrm{H}), 5.60(\mathrm{~s}, 1 \mathrm{H},-\mathrm{OH}), 4.36(\mathrm{dd}, J=10.6,5.9 \mathrm{~Hz}$, $1 \mathrm{H},-\mathrm{OCH}-), 3.82(\mathrm{dd}, J=8.8,6.5 \mathrm{~Hz}, 1 \mathrm{H},-\mathrm{OCH}-), 3.49-3.42(\mathrm{~m}, 1 \mathrm{H},-\mathrm{OCH}-), 3.30$ (dd, $\left.J=9.6,3.5 \mathrm{~Hz}, 1 \mathrm{H},-\mathrm{NH}_{2} \mathrm{CH}-\right), 3.14\left(\mathrm{dd}, J=11.7,7.4 \mathrm{~Hz}, 1 \mathrm{H},-\mathrm{NCH}_{2}-\right), 3.01-2.91(\mathrm{~m}, 1 \mathrm{H}$, $\left.-\mathrm{NCH}_{2}-\right), 2.87(\mathrm{~s}, 6 \mathrm{H},-\mathrm{CH} 3 \times 2), 1.25\left(\mathrm{~s}, 3 \mathrm{H},-\mathrm{CH}_{3}\right), 1.24\left(\mathrm{~s}, 3 \mathrm{H},-\mathrm{CH}_{3}\right), 1.07\left(\mathrm{~s}, 3 \mathrm{H},-\mathrm{CH}_{3}\right)$, $0.94\left(\mathrm{~s}, 3 \mathrm{H},-\mathrm{CH}_{3}\right), 0.86\left(\mathrm{~s}, 3 \mathrm{H},-\mathrm{CH}_{3}\right), 0.81\left(\mathrm{~s}, 3 \mathrm{H},-\mathrm{CH}_{3}\right), 0.73\left(\mathrm{~s}, 3 \mathrm{H},-\mathrm{CH}_{3}\right), 0.72(\mathrm{~s}, 3 \mathrm{H}$, $\left.-\mathrm{CH}_{3}\right) .{ }^{13} \mathrm{C}-\mathrm{NMR}\left(\mathrm{CDCl}_{3}, 100 \mathrm{MHz}\right) \delta(\mathrm{ppm}): 174.52,152.04,134.72,130.34,129.95,129.72$, $128.24,129.64,123.27,118.97,115.20,86.59,85.49,81.96,70.98,70.19,56.03,54.36,52.06$, $50.44,49.45,48.00,45.51,42.32,39.80,38.54,37.92,37.08,34.75,32.70,32.36,31.40,31.28$, $29.79,28.66,28.03,28.01,27.71,26.22,25.09,23.56,18.24,16.51,15.44$. HR-MS (ESI) $m / z$ : calcd. for $\mathrm{C}_{46} \mathrm{H}_{71} \mathrm{~N}_{3} \mathrm{O}_{7} \mathrm{~S}[\mathrm{M}+\mathrm{H}]^{+}:$810.50855, found: 810.50635.

3.3.6. (20S, 24S)-Epoxy-3ß-O-[2-amino-(1-dansylamino)-5-dimethyl amino butyr-yl]-dammarane-12 $\beta, 25$-diol (29)

Yellow solid (yield 69.6\%); m.p. 210.5 211.3 ${ }^{\circ} \mathrm{C} ;[\alpha]_{20}^{D}=84.703$ (c $=0.1, \mathrm{MeOH}: \mathrm{CHCl}_{3}=$ 3:1); ${ }^{1} \mathrm{H}-\mathrm{NMR}(\mathrm{CDCl} 3,400 \mathrm{MHz}) \delta(\mathrm{ppm}): 8.51(\mathrm{~d}, J=8.4 \mathrm{~Hz}, 1 \mathrm{H}, \mathrm{Ar}-\mathrm{H}), 8.27(\mathrm{~d}, J=8.6 \mathrm{~Hz}$, $1 \mathrm{H}, \mathrm{Ar}-\mathrm{H}), 8.22(\mathrm{~d}, J=7.3 \mathrm{~Hz}, 1 \mathrm{H}, \mathrm{Ar}-\mathrm{H}), 7.56(\mathrm{~s}, 1 \mathrm{H}, \mathrm{Ar}-\mathrm{H}), 7.52-7.47(\mathrm{~m}, 1 \mathrm{H}, \mathrm{Ar}-\mathrm{H}), 7.17$ $(\mathrm{d}, J=7.5 \mathrm{~Hz}, 1 \mathrm{H}, \mathrm{Ar}-\mathrm{H}), 5.75(\mathrm{~s}, 1 \mathrm{H},-\mathrm{OH}), 4.38(\mathrm{dd}, J=10.8,5.8 \mathrm{~Hz}, 1 \mathrm{H},-\mathrm{OCH}-), 3.85(\mathrm{dd}$, $J=10.9,5.3 \mathrm{~Hz}, 1 \mathrm{H},-\mathrm{OCH}-), 3.50(\mathrm{td}, J=9.8,4.7 \mathrm{~Hz}, 1 \mathrm{H},-\mathrm{OCH}-), 3.30(\mathrm{dd}, J=9.7,3.5 \mathrm{~Hz}, 1 \mathrm{H}$, $\left.-\mathrm{NH}_{2} \mathrm{CH}-\right), 3.20-3.12\left(\mathrm{~m}, 1 \mathrm{H},-\mathrm{NCH}_{2}-\right), 2.97\left(\mathrm{dd}, J=8.9,4.2 \mathrm{~Hz}, 1 \mathrm{H},-\mathrm{NCH}_{2}-\right), 2.87(\mathrm{~s}, 6 \mathrm{H}$, $-\mathrm{CH} 3 \times 2), 1.26\left(\mathrm{~s}, 3 \mathrm{H},-\mathrm{CH}_{3}\right), 1.23\left(\mathrm{~s}, 3 \mathrm{H},-\mathrm{CH}_{3}\right), 1.08\left(\mathrm{~s}, 3 \mathrm{H},-\mathrm{CH}_{3}\right), 0.98\left(\mathrm{~s}, 3 \mathrm{H},-\mathrm{CH}_{3}\right), 0.88$ $\left(\mathrm{s}, 3 \mathrm{H},-\mathrm{CH}_{3}\right), 0.86\left(\mathrm{~s}, 3 \mathrm{H},-\mathrm{CH}_{3}\right), 0.75\left(\mathrm{~s}, 3 \mathrm{H},-\mathrm{CH}_{3}\right), 0.74\left(\mathrm{~s}, 3 \mathrm{H},-\mathrm{CH}_{3}\right) .13 \mathrm{C}-\mathrm{NMR}\left(\mathrm{CDCl}_{3}\right.$, 
$100 \mathrm{MHz}) \delta$ (ppm): 174.53, 152.06, 135.11, 130.34, 129.97, 129.73, 129.65, 128.25, 123.27, $118.98,115.21,87.47,87.23,81.98,70.52,70.11,56.00,54.44,52.22,50.18,48.96,48.86,45.52$, $39.81,38.22,37.93,37.11,34.70,32.28,31.69,31.33,29.80,28.97,28.61,28.15,27.30,25.12$, $24.30,23.60,18.20,17.84,16.54,16.40,15.52$. HR-MS (ESI) $m / z$ : calcd. for $\mathrm{C}_{46} \mathrm{H}_{71} \mathrm{~N}_{3} \mathrm{O}_{7} \mathrm{~S}$ $[\mathrm{M}+\mathrm{H}]^{+}:$: 10.50855 , found: 810.50653 .

3.3.7. (20S, 24R)-Epoxy-3ß-O-[2-amino-(1-dansylamino)-5-dimethyl amino val-eryl]-dammarane-12 $\beta$, 25-diol (30)

Yellow solid (yield 65.1\%); m.p. $192.5 \sim 192.9^{\circ} \mathrm{C} ;[\alpha]_{20}^{D}=59.357$ (c=0.1, $\mathrm{MeOH}: \mathrm{CHCl}_{3}=$ 3:1); ${ }^{1} \mathrm{H}-\mathrm{NMR}(\mathrm{CDCl} 3,400 \mathrm{MHz}) \delta$ (ppm): 8.50 (d, J = 8.5 Hz, 1H, Ar-H), 8.29 (d, J = 8.7 Hz, $1 \mathrm{H}, \mathrm{Ar}-\mathrm{H}), 8.21$ (dd, J = 7.3, 1.3 Hz, 1H, Ar-H), $7.55-7.50(\mathrm{~m}, 1 \mathrm{H}, \mathrm{Ar}-\mathrm{H}), 7.51-7.46(\mathrm{~m}, 1 \mathrm{H}$, Ar-H), 7.15 (d, J = 7.5 Hz, 1H, Ar-H), 5.57 (s, 1H. -OH), $4.47-4.39$ (m, 1H, -OCH-), 3.82 (dd, $J=8.8,6.6 \mathrm{~Hz}, 1 \mathrm{H},-\mathrm{OCH}-), 3.49$ (td, $J=10.5,4.6 \mathrm{~Hz}, 1 \mathrm{H},-\mathrm{OCH}-), 3.21$ (dd, $J=8.6,4.1 \mathrm{~Hz}$, $\left.1 \mathrm{H},-\mathrm{NH}_{2} \mathrm{CH}-\right), 2.99-2.91\left(\mathrm{~m}, 1 \mathrm{H},-\mathrm{NCH}_{2}-\right), 2.86(\mathrm{~s}, 6 \mathrm{H},-\mathrm{CH} 3 \times 2), 2.81$ (ddd, $J=12.7$, 7.5, $\left.5.4 \mathrm{~Hz}, 1 \mathrm{H},-\mathrm{NCH}_{2}-\right), 1.25\left(\mathrm{~s}, 3 \mathrm{H},-\mathrm{CH}_{3}\right), 1.24\left(\mathrm{~s}, 3 \mathrm{H},-\mathrm{CH}_{3}\right), 1.07\left(\mathrm{~s}, 3 \mathrm{H},-\mathrm{CH}_{3}\right), 0.96$ $\left(\mathrm{s}, 3 \mathrm{H},-\mathrm{CH}_{3}\right), 0.87\left(\mathrm{~s}, 3 \mathrm{H},-\mathrm{CH}_{3}\right), 0.84\left(\mathrm{~s}, 3 \mathrm{H},-\mathrm{CH}_{3}\right), 0.77\left(\mathrm{~s}, 3 \mathrm{H},-\mathrm{CH}_{3}\right), 0.74\left(\mathrm{~s}, 3 \mathrm{H},-\mathrm{CH}_{3}\right)$. ${ }^{13} \mathrm{C}-\mathrm{NMR}\left(\mathrm{CDCl}_{3}, 100 \mathrm{MHz}\right) \delta$ (ppm): 175.30, 152.02, 134.97, 130.27, 129.94, 129.67, 129.64, $128.21,123.30,118.95,115.19,86.59,85.49,81.78,71.00,70.20,56.06,54.32,52.07,50.46,49.45$, $48.00,45.52,43.15,39.81,38.61,38.02,37.11,34.77,32.70,32.25,31.41,28.66,28.14,28.01$, $27.71,26.36,26.23,25.09,23.75,18.25,16.63,16.45,15.45$. HR-MS (ESI) $m / z$ : calcd. for C47H73N3O7S $[\mathrm{M}+\mathrm{H}]^{+}:$824.52420, found: 824.52173 .

3.3.8. (20S, 24S)-Epoxy-3ß-O-[2-amino-(1-dansylamino)-5-dimethyl amino val-eryl]-dammarane-12 $\beta$, 25-diol (31)

Yellow solid (yield 63.4\%); m.p. 198.2 199.3 ${ }^{\circ} \mathrm{C} ;[\alpha]_{20}^{D}=48.687$ (c = 0.1, MeOH:CHCl $3=$ 3:1); ${ }^{1} \mathrm{H}-\mathrm{NMR}\left(\mathrm{CDCl}_{3}, 400 \mathrm{MHz}\right) \delta(\mathrm{ppm}): 8.51(\mathrm{~d}, J=10.6 \mathrm{~Hz}, 1 \mathrm{H}, \mathrm{Ar}-\mathrm{H}), 8.29(\mathrm{~d}, J=8.7 \mathrm{~Hz}$, $1 \mathrm{H}, \mathrm{Ar}-\mathrm{H}), 8.24-8.19(\mathrm{~m}, 1 \mathrm{H}, \mathrm{Ar}-\mathrm{H}), 7.58-7.52(\mathrm{~m}, 1 \mathrm{H}, \mathrm{Ar}-\mathrm{H}), 7.53-7.47$ (m, 1H, Ar-H), $7.16(\mathrm{~d}, J=8.3 \mathrm{~Hz}, 1 \mathrm{H}, \mathrm{Ar}-\mathrm{H}), 5.75(\mathrm{~s}, 1 \mathrm{H},-\mathrm{OH}), 4.44$ (dd, J = 9.3, 7.2 Hz, 1H, -OCH-), $3.86(\mathrm{dd}, J=10.8,5.4 \mathrm{~Hz}, 1 \mathrm{H},-\mathrm{OCH}-), 3.51$ (td, $J=10.3,4.8 \mathrm{~Hz}, 1 \mathrm{H},-\mathrm{OCH}-), 3.23$ (dd, $\left.J=8.7,4.1 \mathrm{~Hz}, 1 \mathrm{H},-\mathrm{NH}_{2} \mathrm{CH}-\right), 3.01-2.91\left(\mathrm{~m}, 1 \mathrm{H},-\mathrm{NCH}_{2}-\right), 2.87(\mathrm{~s}, 6 \mathrm{H},-\mathrm{CH} 3 \times 2), 2.80$ $\left(\mathrm{ddd}, J=12.7,7.6,5.3 \mathrm{~Hz}, 1 \mathrm{H},-\mathrm{NCH}_{2}-\right), 1.26\left(\mathrm{~s}, 3 \mathrm{H},-\mathrm{CH}_{3}\right), 1.23\left(\mathrm{~s}, 3 \mathrm{H},-\mathrm{CH}_{3}\right), 1.08(\mathrm{~s}, 3 \mathrm{H}$, $\left.-\mathrm{CH}_{3}\right), 0.99\left(\mathrm{~s}, 3 \mathrm{H},-\mathrm{CH}_{3}\right), 0.89\left(\mathrm{~s}, 3 \mathrm{H},-\mathrm{CH}_{3}\right), 0.88\left(\mathrm{~s}, 3 \mathrm{H},-\mathrm{CH}_{3}\right), 0.79\left(\mathrm{~s}, 3 \mathrm{H},-\mathrm{CH}_{3}\right), 0.75(\mathrm{~s}$, $\left.3 \mathrm{H},-\mathrm{CH}_{3}\right) .{ }^{13} \mathrm{C}-\mathrm{NMR}\left(\mathrm{CDCl}_{3}, 100 \mathrm{MHz}\right) \delta(\mathrm{ppm}): 175.26,152.04,134.96,130.28,129.96$, $129.68,129.66,128.22,123.31,118.95,115.20,87.46,87.23,81.81,70.53,70.13,56.03,54.33$, $52.23,50.19,48.97,48.86,45.52,43.15,39.81,38.04,37.13,34.72,32.29,32.02,31.69,31.3$, $29.79,28.96,28.62,28.13,26.39,25.13,24.30,23.78,18.23,17.85,16.67,16.41,15.54$. HR-MS (ESI) $m / z$ : calcd. for $\mathrm{C}_{47} \mathrm{H}_{73} \mathrm{~N}_{3} \mathrm{O}_{7} \mathrm{~S}[\mathrm{M}+\mathrm{H}]^{+}:$: 24.52420 , found: 824.52191 .

3.3.9. (20S, 24R)-Epoxy-3 $\beta-O-\left[3-p-\left(N^{\prime}-F m o c\right)\right.$ aromatic ring-2-alanine]-dammarane-12 $\beta$, 25-diol (34)

White solid (yield 73.2\%); m.p. $128.4 \sim 129.5^{\circ} \mathrm{C} ;[\alpha]_{20}^{D}=16.0(\mathrm{c}=0.1, \mathrm{MeOH}: \mathrm{CHCl3}=3: 1)$; ${ }^{1} \mathrm{H}-\mathrm{NMR}\left(\mathrm{CDCl}_{3}, 400 \mathrm{MHz}\right) \delta(\mathrm{ppm}): 7.75(\mathrm{~d}, J=7.5 \mathrm{~Hz}, 2 \mathrm{H}, \mathrm{Ar}-\mathrm{H}), 7.59(\mathrm{~d}, J=7.4 \mathrm{~Hz}, 2 \mathrm{H}$, Ar-H), $7.38(\mathrm{t}, J=7.4 \mathrm{~Hz}, 2 \mathrm{H}, \mathrm{Ar}-\mathrm{H}), 7.29(\mathrm{t}, J=7.4 \mathrm{~Hz}, 4 \mathrm{H}, \mathrm{Ar}-\mathrm{H}), 7.12(\mathrm{~d}, J=8.0 \mathrm{~Hz}, 2 \mathrm{H}$, Ar-H), 6.99 (s, 1H, -NH-CO-), 4.49 (d, J = 6.4 Hz, 3H, -OCH-, - $\mathrm{CH}_{2}-$ ), 4.24 (t, J = 6.6 Hz, 1H, -OCH-), 3.83 (dd, J = 8.6, $6.6 \mathrm{~Hz}, 1 \mathrm{H},-\mathrm{OCH}-), 3.50$ (td, $J=10.4,4.4 \mathrm{~Hz}, 1 \mathrm{H},-\mathrm{CH}-), 2.97$ $\left(\mathrm{m}, 2 \mathrm{H},-\mathrm{CH}_{2}-\right), 1.26\left(\mathrm{~s}, 3 \mathrm{H},-\mathrm{CH}_{3}\right), 1.24\left(\mathrm{~s}, 3 \mathrm{H},-\mathrm{CH}_{3}\right), 1.08\left(\mathrm{~s}, 3 \mathrm{H},-\mathrm{CH}_{3}\right), 0.96\left(\mathrm{~s}, 3 \mathrm{H},-\mathrm{CH}_{3}\right)$, $0.87\left(\mathrm{~s}, 3 \mathrm{H},-\mathrm{CH}_{3}\right), 0.85\left(\mathrm{~s}, 3 \mathrm{H},-\mathrm{CH}_{3}\right), 0.80(\mathrm{~s}, 6 \mathrm{H},-\mathrm{CH} 3 \times 2) .{ }^{13} \mathrm{C}-\mathrm{NMR}\left(\mathrm{CDCl}_{3}, 100 \mathrm{MHz}\right) \delta$ (ppm): 143.75, 141.32, 130.02, 127.75, 127.11, 124.97, 120.01, 86.49, 85.38, 82.11, 70.92, 70.16, $66.78,56.02,55.32,51.99,50.38,49.35,47.94,47.11,39.74,38.56,37.94,37.02,34.71,32.59$, 31.33, 31.18, 29.30, 28.57, 27.99, 27.92, 27.56, 26.10, 25.00, 23.57, 18.14, 16.44, 16.36, 15.37 . HR-MS (ESI) $m / z$ : calcd. for $\mathrm{C}_{54} \mathrm{H}_{72} \mathrm{~N}_{2} \mathrm{O}_{7}[\mathrm{M}+\mathrm{H}]^{+}:$: 861.5412, found: 861.53870 . 


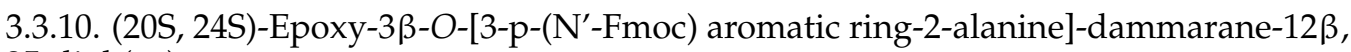
25-diol (35)

White solid (yield 73.2\%); m.p. 135.4 135. $6^{\circ} \mathrm{C} ;[\alpha]_{20}^{D}=6.0$ (c = 0.1, $\mathrm{MeOH}: \mathrm{CHCl}_{3}=3: 1$ ); ${ }^{1} \mathrm{H}-\mathrm{NMR}\left(\mathrm{CDCl}_{3}, 400 \mathrm{MHz}\right) \delta(\mathrm{ppm}): 7.76(\mathrm{~d}, J=7.5 \mathrm{~Hz}, 2 \mathrm{H}, \mathrm{Ar}-\mathrm{H}), 7.60(\mathrm{~d}, J=7.5 \mathrm{~Hz}, 2 \mathrm{H}$, Ar-H), $7.39(\mathrm{t}, J=7.4 \mathrm{~Hz}, 2 \mathrm{H}, \mathrm{Ar}-\mathrm{H}), 7.30(\mathrm{td}, J=7.5,1.1 \mathrm{~Hz}, 4 \mathrm{H}, \mathrm{Ar}-\mathrm{H}), 7.12(\mathrm{~d}, J=8.4 \mathrm{~Hz}$, $2 \mathrm{H}, \mathrm{Ar}-\mathrm{H}), 6.81(\mathrm{~s}, 1 \mathrm{H},-\mathrm{NH}-\mathrm{CO}-), 5.74(\mathrm{~s}, 1 \mathrm{H},-\mathrm{OH}), 4.55-4.45\left(\mathrm{~m}, 3 \mathrm{H},-\mathrm{NH}-,-\mathrm{CH}_{2}-\right), 4.25(\mathrm{t}$, $J=6.6 \mathrm{~Hz}, 1 \mathrm{H},-\mathrm{OCH}-), 3.86(\mathrm{dd}, J=10.8,5.4 \mathrm{~Hz}, 1 \mathrm{H},-\mathrm{OCH}-), 3.52(\mathrm{td}, J=10.4,4.8 \mathrm{~Hz}, 1 \mathrm{H}$, - $\mathrm{CH}-), 2.92\left(\mathrm{ddd}, \mathrm{J}=81.4,13.8,6.4 \mathrm{~Hz}, 2 \mathrm{H},-\mathrm{CH}_{2}-\right), 1.26\left(\mathrm{~s}, 3 \mathrm{H},-\mathrm{CH}_{3}\right), 1.22\left(\mathrm{~s}, 3 \mathrm{H},-\mathrm{CH}_{3}\right)$, $1.09\left(\mathrm{~s}, 3 \mathrm{H},-\mathrm{CH}_{3}\right), 1.00(\mathrm{~s}, 3 \mathrm{H},-\mathrm{CH} 3), 0.89(\mathrm{~s}, 6 \mathrm{H},-\mathrm{CH} 3 \times 2), 0.83\left(\mathrm{~s}, 3 \mathrm{H},-\mathrm{CH}_{3}\right), 0.82(\mathrm{~s}, 3 \mathrm{H}$, - $\left.\mathrm{CH}_{3}\right) .{ }^{13} \mathrm{C}-\mathrm{NMR}\left(\mathrm{CDCl}_{3}, 100 \mathrm{MHz}\right) \delta$ (ppm): 174.55, 143.76, 141.36, 129.99, 127.77, 127.12, $124.95,120.04,87.15,81.76,70.48,70.09,66.80,56.02,55.71,52.16,50.15,48.94,48.83,47.16$, $40.12,39.78,38.56,37.98,37.09,34.69,32.21,31.64,29.70,28.89,28.54,28.03,25.10,24.24$, $23.64,18.19,17.77,16.50,16.35,15.48$. HR-MS (ESI) $m / z$ : calcd. for $\mathrm{C}_{54} \mathrm{H}_{72} \mathrm{~N}_{2} \mathrm{O}_{7}[\mathrm{M}+\mathrm{H}]^{+}$: 861.5412, found: 861.53802 .

\subsection{Antibacterial Activity}

HA-MRSA 18-19, 18-20, and S. aureus ATCC 29213 were used to evaluate the antibacterial activity of all the compounds. HA-MRSA 18-19 and 18-20 were hospital-acquired MRSA strains (Chengdu, China). S. aureus ATCC 29213 was used as a control. MuellerHinton agar was prepared, with the final concentrations of the drug in each plate ranging from 128 to $0.008 \mu \mathrm{g} / \mathrm{mL}$. Then, approximately $10^{4}$ colony-forming units (CFUs) of diluted bacterial solution were inoculated onto the prepared Mueller-Hinton agar. Then, HA-MRSA strains 18-15, 18-19, and 18-20 were used to evaluate selected compounds in a secondary assay.

\subsection{Synergistic Antibacterial Activity}

HA-MRSA 18-15 was used to evaluate the synergistic antibacterial activity of the selected compounds. First, the compounds were serially diluted from 32 to 1/32 times their MIC value and then a total of $100 \mu \mathrm{L}$ of a mixed solution containing two compounds were added to the columns and rows of a 96-well plate. Then, $100 \mu \mathrm{L}$ of a diluted bacterial solution containing approximately $5 \times 10^{4} \mathrm{CFU}$ were added to each well. The results were recorded after cultivation at $37^{\circ} \mathrm{C}$ for $24 \mathrm{~h}$. A $20 \mu \mathrm{L}$ sample of the mixed solution from the 96-well plate was spread on drug-free Mueller-Hinton agar, and after cultivation at $37^{\circ} \mathrm{C}$ for $24 \mathrm{~h}$, the minimum bactericidal concentration (MBC) effect was determined.

\section{Conclusions}

Natural products are irreplaceable in the development of new drugs. Our previous research showed that ocotillol-type derivatives have good antibacterial activity against community-acquired MRSA (CA-MRSA). Recent research showed that ocotillol-type ginsenosides also have good antibacterial activity against HA-MRSA. In this research, ocotilloltype derivatives with nitrogen-containing groups were synthesized and their antibacterial activity was evaluated toward HA-MRSA in vitro. Compounds 20-23 and 28-31 exhibited potent antibacterial activity with MICs of 1-64 $\mu \mathrm{g} / \mathrm{mL}$. The results suggested that ocotilloltype compounds coupled with $\mathrm{N}$-heterocycles containing a tertiary amine did not have improved antibacterial activity over ocotillol-type ginsenosides, and ocotillol-type derivatives with a connecting Fmoc were better than those with a connecting dansyl chloride. Compound 20 showed good antibacterial activity with an MIC of $4 \mu \mathrm{g} / \mathrm{mL}$, and synergistic inhibitory effects when combined with conventional antibiotics CHL or KAN as shown by the FICI values of 0.5 and 0.3125 , respectively. Compound 21 showed better antibacterial activity than compound 20 with an MIC of $1 \mu \mathrm{g} / \mathrm{mL}$, and synergistic inhibitory effects when combined with CHL or KAN as shown by the FICI value of 0.375 . The mechanism(s) responsible for the antibacterial and synergistic antibacterial effects of ocotillol-type derivatives against HA-MRSA strains has been inadequately studied. Our previous work showed that ocotillol-type derivatives were mainly distributed on the bacterial cell membrane; thus, 
the mechanism of the antibacterial potency and synergistic effect of ocotillol derivatives is probably related to the bacterial cell membrane. Compound $\mathbf{2 1}$ is a good probe that can be used to investigate the antibacterial and synergistic antibacterial mechanisms of ocotillol antibacterial derivatives.

Supplementary Materials: Supplementary materials are available online.

Author Contributions: Conceptualization, Y.B. and Q.M.; methodology, K.W.; validation, D.Z., Y.C. and Z.S.; data curation, Q.M. and R.W.; supervision, Y.B.; writing-original draft preparation, D.Z., Y.C. and Y.B.; All authors have read and agreed to the published version of the manuscript.

Funding: This work was funded by the National Natural Science Foundation of China (81773563), The Science and Technology Support Program for Youth Innovation in Universities of Shandong (2020KJM003).

Institutional Review Board Statement: Not applicable.

Informed Consent Statement: Not applicable.

Data Availability Statement: Data are contained within the article and the supplementary material.

Acknowledgments: The authors are thankful to Sichuan Industrial Institute of Antibiotics for help in testing antibacterial activity of ocotillol derivatives.

Conflicts of Interest: The authors declare no conflict of interest.

Sample Availability: Samples of the compounds are available from the authors.

\section{References}

1. Gilbert, J.A.; Blaser, M.J.; Caporaso, J.G.; Jansson, J.K.; Lynch, S.V.; Knight, R. Current understanding of the human microbiome. Nat. Med. 2018, 24, 392-400. [CrossRef]

2. Schwabe, R.F.; Jobin, C. The microbiome and cancer. Nat. Rev. Cancer 2013, 13, 800-812. [CrossRef]

3. McEwen, S.A.; Collignon, P.J. Antimicrobial Resistance: A One Health Perspective. Microbiol. Spectr. 2018, 6, 26. [CrossRef]

4. Ferri, M.; Ranucci, E.; Romagnoli, P.; Giaccone, V. Antimicrobial resistance: A global emerging threat to public health systems. Crit. Rev. Food Sci. Nutr. 2017, 57, 2857-2876. [CrossRef]

5. Hoiby, N.; Bjarnsholt, T.; Givskov, M.; Molin, S.; Ciofu, O. Antibiotic resistance of bacterial biofilms. Int. J. Antimicrob. Agents 2010, 35, 322-332. [CrossRef] [PubMed]

6. Tacconelli, E.; Carrara, E.; Savoldi, A.; Harbarth, S.; Mendelson, M.; Monnet, D.L.; Pulcini, C.; Kahlmeter, G.; Kluytmans, J.; Carmeli, Y.; et al. Discovery, research, and development of new antibiotics: The WHO priority list of antibiotic-resistant bacteria and tuberculosis. Lancet Infect. Dis. 2018, 18, 318-327. [CrossRef]

7. Newman, D.J.; Cragg, G.M. Natural Products as Sources of New Drugs over the Nearly Four Decades from 01/1981 to 09/2019. J. Nat. Prod. 2020, 83, 770-803. [CrossRef] [PubMed]

8. Newman, D.J.; Cragg, G.M. Natural Products as Sources of New Drugs from 1981 to 2014. J. Nat. Prod. 2016, 79 , 629-661. [CrossRef] [PubMed]

9. Farhadi, F.; Khameneh, B.; Iranshahi, M.; Iranshahy, M. Antibacterial activity of flavonoids and their structure-activity relationship: An update review. Phytother. Res. 2019, 33, 13-40. [CrossRef]

10. Langeveld, W.T.; Veldhuizen, E.J.; Burt, S.A. Synergy between essential oil components and antibiotics: A review. Crit. Rev. Microbiol. 2014, 40, 76-94. [CrossRef] [PubMed]

11. Cushnie, T.P.; Lamb, A.J. Recent advances in understanding the antibacterial properties of flavonoids. Int. J. Antimicrob. Agents 2011, 38, 99-107. [CrossRef]

12. Oloyede, H.O.B.; Ajiboye, H.O.; Salawu, M.O.; Ajiboye, T.O. Influence of oxidative stress on the antibacterial activity of betulin, betulinic acid and ursolic acid. Microb. Pathog. 2017, 111, 338-344. [CrossRef]

13. Tong, S.Y.C.; Davis, J.S.; Eichenberger, E.; Holland, T.L.; Fowler, V.G. Staphylococcus aureus Infections: Epidemiology, Pathophysiology, Clinical Manifestations, and Management. Clin. Microbiol. Rev. 2015, 28, 603-661. [CrossRef]

14. Chung, P.Y.; Navaratnam, P.; Chung, L.Y. Synergistic antimicrobial activity between pentacyclic triterpenoids and antibiotics against Staphylococcus aureus strains. Ann. Clin. Microbiol. Antimicrob. 2011, 10. [CrossRef]

15. Ayaz, M.; Ullah, F.; Sadiq, A.; Ullah, F.; Ovais, M.; Ahmed, J.; Devkota, H.P. Synergistic interactions of phytochemicals with antimicrobial agents: Potential strategy to counteract drug resistance. Chem. Biol. Interact. 2019, 308, 294-303. [CrossRef]

16. Cappiello, F.; Loffredo, M.R.; Del Plato, C.; Cammarone, S.; Casciaro, B.; Quaglio, D.; Mangoni, M.L.; Botta, B.; Ghirga, F. The Revaluation of Plant-Derived Terpenes to Fight Antibiotic-Resistant Infections. Antibiotics 2020, 9, 325. [CrossRef]

17. Wolska, K.I.; Grudniak, A.M.; Fiecek, B.; Kraczkiewicz-Dowjat, A.; Kurek, A. Antibacterial activity of oleanolic and ursolic acids and their derivatives. Cent. Eur. J. Biol. 2010, 5, 543-553. [CrossRef] 
18. Kim, J.H. Pharmacological and medical applications of Panax ginseng and ginsenosides: A review for use in cardiovascular diseases. J. Ginseng Res. 2018, 42, 264-269. [CrossRef] [PubMed]

19. Kim, J.H.; Yi, Y.S.; Kim, M.Y.; Cho, J.Y. Role of ginsenosides, the main active components of Panax ginseng, in inflammatory responses and diseases. J. Ginseng Res. 2017, 41, 435-443. [CrossRef] [PubMed]

20. Lee, C.H.; Kim, J.H. A review on the medicinal potentials of ginseng and ginsenosides on cardiovascular diseases. J. Ginseng Res. 2014, 38, 161-166. [CrossRef] [PubMed]

21. Liu, J.; Xu, Y.; Yang, J.; Wang, W.; Zhang, J.; Zhang, R.; Meng, Q. Discovery, semisynthesis, biological activities, and metabolism of ocotillol-type saponins. J. Ginseng Res. 2017, 41, 373-378. [CrossRef]

22. Bi, Y.; Ma, C.; Zhang, H.; Zhou, Z.; Yang, J.; Zhang, Z.; Meng, Q.; Lewis, P.J.; Xu, J. Novel 3-substituted ocotillol-type triterpenoid derivatives as antibacterial candidates. Chem. Biol. Drug Des. 2014, 84, 489-496. [CrossRef] [PubMed]

23. Bi, Y.; Yang, X.; Zhang, T.T.; Liu, Z.Y.; Zhang, X.C.; Lu, J.; Cheng, K.G.; Xu, J.Y.; Wang, H.B.; Lv, G.Y.; et al. Design, synthesis, nitric oxide release and antibacterial evaluation of novel nitrated ocotillol-type derivatives. Eur. J. Med. Chem. 2015, 101, 71-80. [CrossRef] [PubMed]

24. Bi, Y.; Ma, C.; Zhou, Z.; Zhang, T.; Zhang, H.; Zhang, X.; Lu, J.; Meng, Q.; Lewis, P.; Xu, J. Synthesis and Antibacterial Evaluation of Novel Hydrophilic Ocotillol-Type Triterpenoid Derivatives from 20(S)-Protopanaxadiol. Rec. Nat. Prod. 2015, 9, 356-368.

25. Cao, Y.C.; Wang, K.Y.; Wang, J.L.; Cheng, H.R.; Ma, M.X.; Meng, Q.G.; Li, X.P.; Bi, Y. Design, synthesis and antibacterial evaluation of ocotillol derivatives with polycyclic nitrogen-containing groups. Future Med. Chem. 2021, 13. [CrossRef]

26. Bi, Y.; Liu, X.X.; Zhang, H.Y.; Yang, X.; Liu, Z.Y.; Lu, J.; Lewis, P.J.; Wang, C.Z.; Xu, J.Y.; Meng, Q.G.; et al. Synthesis and Antibacterial Evaluation of Novel 3-Substituted Ocotillol-Type Derivatives as Leads. Molecules 2017, 22, 590. [CrossRef] [PubMed]

27. Cao, Y.C.; Wang, K.Y.; Xu, S.; Kong, L.T.; Bi, Y.; Li, X.P. Recent Advances in the Semisynthesis, Modifications and Biological Activities of Ocotillol-Type Triterpenoids. Molecules 2020, 25, 5562. [CrossRef]

28. Ge, X.; Xu, Z. 1,2,4-Triazole hybrids with potential antibacterial activity against methicillin-resistant Staphylococcus aureus. Arch. Pharm. 2021, 354, e2000223. [CrossRef] [PubMed]

29. Santos, F.R.S.; Andrade, J.T.; Sousa, C.D.F.; Fernandes, J.S.; Carmo, L.F.; Araujo, M.G.F.; Ferreira, J.M.S.; Villar, J. Synthesis and Evaluation of the in vitro Antimicrobial Activity of Triazoles, Morpholines and Thiosemicarbazones. Med. Chem. 2019, 15, 38-50. [CrossRef]

30. Yin, J.; Kwon, Y.; Kim, D.; Lee, D.; Kim, G.; Hu, Y.; Ryu, J.H.; Yoon, J. Preparation of a cyanine-based fluorescent probe for highly selective detection of glutathione and its use in living cells and tissues of mice. Nat. Protoc. 2015, 10, 1742-1754. [CrossRef]

31. Lin, G.J.; Luo, S.P.; Zheng, X.; Ye, J.L.; Huang, P.Q. Enantiodivergent synthesis of trans-3,4-disubstituted succinimides by SmI(2)-mediated Reformatsky-type reaction. Tetrahedron Lett. 2008, 49, 4007-4010. [CrossRef]

32. Kim, J.-H.; Kim, N.-H.; Kim, E.-J.; Kim, J.H.; Lee, M.-Y.; Park, Y.-H.; Lee, J.R.; Park, S.-C.; Jang, M.-K. Antibacterial effects of amino acids-grafted water-soluble chitosan against drug-resistant bacteria. Biotechnol. Bioprocess Eng. 2016, 21, 183-189. [CrossRef]

33. Gahane, A.Y.; Ranjan, P.; Singh, V.; Sharma, R.K.; Sinha, N.; Sharma, M.; Chaudhry, R.; Thakur, A.K. Fmoc-phenylalanine displays antibacterial activity against Gram-positive bacteria in gel and solution phases. Soft Matter 2018, 14, 2234-2244. [CrossRef] [PubMed]

34. Miao, J.; Chen, L.; Wang, J.; Wang, W.; Chen, D.; Li, L.; Li, B.; Deng, Y.; Xu, Z. Current methodologies on genotyping for nosocomial pathogen methicillin-resistant Staphylococcus aureus (MRSA). Microb. Pathog. 2017, 107, 17-28. [CrossRef] [PubMed]

35. Hassoun, A.; Linden, P.K.; Friedman, B. Incidence, prevalence, and management of MRSA bacteremia across patient populations-a review of recent developments in MRSA management and treatment. Crit. Care 2017, 21, 211. [CrossRef] [PubMed]

36. Stefani, S.; Chung, D.R.; Lindsay, J.A.; Friedrich, A.W.; Kearns, A.M.; Westh, H.; Mackenzie, F.M. Meticillin-resistant Staphylococcus aureus (MRSA): Global epidemiology and harmonisation of typing methods. Int. J. Antimicrob. Agents 2012, 39, $273-282$. [CrossRef] [PubMed]

37. Zhou, Z.; Ma, C.; Zhang, H.; Bi, Y.; Chen, X.; Tian, H.; Xie, X.; Meng, Q.; Lewis, P.J.; Xu, J. Synthesis and biological evaluation of novel ocotillol-type triterpenoid derivatives as antibacterial agents. Eur. J. Med. Chem. 2013, 68, 444-453. [CrossRef]

38. Kunnumakkara, A.B.; Bordoloi, D.; Padmavathi, G.; Monisha, J.; Roy, N.K.; Prasad, S.; Aggarwal, B.B. Curcumin, the golden nutraceutical: Multitargeting for multiple chronic diseases. Br. J. Pharmacol. 2017, 174, 1325-1348. [CrossRef] 